\title{
Space discontinuous Galerkin method for shallow water flows — kinetic and HLLC flux, and potential vorticity generation
}

\author{
P. A. Tassi, ${ }^{\mathrm{a}, \mathrm{b}}$ O. Bokhove, ${ }^{\mathrm{a}, *}$ \& C.A.Vionnet ${ }^{\mathrm{b}}$

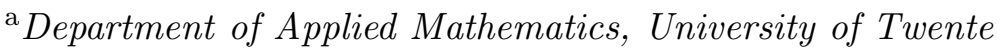 \\ P.O. Box 217, 7500 AE, Enschede, The Netherlands \\ ${ }^{\mathrm{b}}$ Department of Engineering and Water Resources, Universidad Nacional del \\ Litoral \& Conicet, Santa Fe, Argentina
}

\begin{abstract}
In this paper, a second order space discontinuous Galerkin (DG) method is presented for the numerical solution of inviscid shallow water flows over varying bottom topography. Novel in the implementation is the use of HLLC and kinetic numerical fluxes ${ }^{1}$ in combination with a dissipation operator, applied only locally around discontinuities to limit spurious numerical oscillations. Numerical solutions over (non-)uniform meshes are verified against exact solutions; the numerical error in the $L_{2}$-norm and the convergence of the solution are computed. Bore-vortex interactions are studied analytically and numerically to validate the model; these include bores as "breaking waves" in a channel and a bore traveling over a conical and Gaussian hump. In these complex numerical test cases, we correctly predict the generation of potential vorticity by non-uniform bores. Finally, we successfully validate the numerical model against measurements of steady oblique hydraulic jumps in a channel with a contraction. In the latter case, the kinetic flux is shown to be more robust.
\end{abstract}

Key words: Discontinuous Galerkin, Finite elements, Shallow water flows, Discontinuity detector, Bore-vortex interactions, Potential vorticity generation

\footnotetext{
$\overline{1}$ HLLC stands for the Harten, Lax, and Van Leer or HLL flux extended to deal with Contact waves, whence the abbreviation HLLC.

* Corresponding authors: Dr. Onno Bokhove, o.bokhove@math.utwente.nl ; Pablo Tassi, M.Eng., p.a.tassi@math.utwente.nl
} 


\section{Introduction}

Many river and estuarine flows are three-dimensional (3D), highly variable in time and well-described by the conservation laws of mass and momentum without the need to resort to the more complex situation of a density-stratified fluid. Furthermore, it is often sufficient to consider a restricted form of the Euler equations for an incompressible, homogeneous fluid, namely its hydrostatic, depth-averaged form, which is $2 \mathrm{D}$ in the horizontal plane [39]. The resulting, so-called shallow water equations (SWE) are often used as leading order model for river and shallow lake hydrodynamics, floodplain flows, coastal circulation as well as long wave run-up, among other cases of engineering and scientific interest.

Several numerical algorithms have been developed over the years to solve the SWE approximately with finite difference, finite volume and finite element methods. Early numerical experiments showed, however, that when the discrete finite element problem is formulated by approximating the depthaveraged velocity $\mathbf{u}$ and the water depth $h$ in the SWE with an inappropriate choice of interpolation functions, the solution does not converge as the mesh is refined. In some instances, so-called "checker-board modes" arise in such a way that large oscillations enter into the computed solution [40]. The use of non-staggered grids in a finite difference setting or the straightforward use of equal-order interpolation spaces in a finite element formulation thus often lead to spurious modes. These modes can be suppressed through the use of staggered grids [39], variables suited to potential flow, or with mixed interpolation spaces that satisfy a consistency condition [40]. The other successful techniques in removing spurious oscillation modes from the solution to the SWE that received considerable attention in recent years are the quasi-bubble approximation and the Generalized Wave Continuity Equation method (GWCE). The GWCE manipulates the primitive form of the SWE prior to its approximation within the finite element framework, and possesses a monotonic dispersion relationship that does not inherit a second artificial high wave number associated with spurious modes [4]. On the other hand, the quasi-bubble approach adds velocity nodes at the centre of the linear space used to approximate the water depth. Nevertheless, a numerical scheme based on equal order approximations procedure is considered to be more efficient from an implementation point of view [22], [42], [12]. Such a scheme is considered in this paper.

In the last few years, the discontinuous Galerkin (DG) method merged ideas from high-resolution finite difference and finite volume schemes for solving nonlinear hyperbolic systems within a finite element framework [15]. In the DG formulation, locally continuous basis and trial functions are introduced, which are globally discontinuous, within each element. Added advantages of DG finite-element methods are such that it is relatively easy [14]: (i) to im- 
prove the order of accuracy, thus allowing efficient $p$-adaptivity; (ii) to refine the grid locally, without taking into account the continuity restrictions typical of conforming finite element methods, thus allowing efficient $h$-adaptivity; and, (iii) to perform parallel computations [16], since the method is extremely local in data communication. However, the overall formulation involves more degrees of freedom in comparison with finite volume and finite difference methods. For more details and extensions to DG methods with moving boundaries in compressible and shallow water flows, we also refer to Cockburn and Shu [13], Van der Vegt and Van der Ven [38], Bokhove [6] and Bokhove et al. [8], and references therein.

This paper is concerned with the design of a DG finite-element approximation for inviscid SWE. Novel in this work are: I) the use of numerical fluxes in combination with a dissipation operator, applied only locally around discontinuities to limit spurious numerical oscillations; II) the derivation and application of a kinetic numerical flux as alternative to existent numerical fluxes such as the HLLC numerical flux; III) the study of the generation of potential vorticity (PV) anomalies due to bore-vortex interactions in shallow water flows; and, IV) a validation of the numerical model against laboratory data of oblique hydraulic jumps for flow in a flume with a contraction. Bores in shallow water flows are discontinuities in the water depth and akin to shocks, or discontinuities in the density, in gas dynamics. In contrast to flow gas dynamics, energy is dissipated in the bores and hydraulic jumps in the SWE, while mass and (locally) momentum are conserved across such discontinuities. The original shallow water model for smooth flows is therefore extended to include these bores, where energy is dissipated such that multivalued solutions only occur as jumps. In two horizontal dimensions these jumps occur along (time dependent) line discontinuities, and in one dimension at isolated (time dependent) points. These simulations of bore-vortex interaction form demanding test cases, especially for the numerical dissipation around bores, which is unfortunately less well-known in the CFD community. The generation of PV by bores has a counterpart in compressible flow as the generation of Ertel's PV and entropy by shocks, implying that our bore-vortex tests have wider applicability. Furthermore, our numerical verification confirms the stability and efficiency of the DG method.

We thus consider a formulation of the SWE properly allowing for hydraulic jumps and bores as a simple (asymptotic) model of wave breaking. Consequently, we do not use a potential flow formulation, which is more stable but only valid in wave propagation outside regions with shoaling and wave breaking.

The outline of the paper is as follows. We introduce the SWE in Section 2 in dimensional and non-dimensional form, and provide a simplified exposition of potential vorticity generation by bores. The DG spatial discretization is 
presented in Section 3 for the SWE, including a presentation of HLLC and kinetic numerical fluxes. In Section 4, the numerical scheme is verified by comparing simulations over (non-)uniform grids with exact one-dimensional solutions. The model is validated by analyzing bore-vortex interactions in a channel and over conical and Gaussian topography, and against laboratory measurements of oblique hydraulic jumps in Section 5. Conclusions are drawn in Section 6.

\section{Governing Equations}

The SWE in conservative form read (see, e.g., [29])

$$
\begin{aligned}
\partial_{t^{*}} h^{*}+\partial_{x^{*}}\left(h^{*} u^{*}\right)+\partial_{y^{*}}\left(h^{*} v^{*}\right) & =0, \\
\partial_{t^{*}}\left(h^{*} u^{*}\right)+\partial_{x^{*}}\left(h^{*} u^{* 2}+g^{*} h^{* 2} / 2\right)+\partial_{y^{*}}\left(h^{*} u^{*} v^{*}\right) & =-g^{*} h^{*} \partial_{x^{*}} h_{b}^{*}, \\
\partial_{t^{*}}\left(h^{*} v^{*}\right)+\partial_{x^{*}}\left(h^{*} u^{*} v^{*}\right)+\partial_{y^{*}}\left(h^{*} v^{* 2}+g^{*} h^{* 2} / 2\right) & =-g^{*} h^{*} \partial_{y^{*}} h_{b}^{*},
\end{aligned}
$$

where partial derivatives are denoted by $\partial_{t^{*}}=\partial / \partial t^{*}$ and so forth; $\mathbf{u}^{*}\left(\mathbf{x}^{*}, t\right)=$ $\left(u^{*}\left(\mathbf{x}^{*}, t\right), v^{*}\left(\mathbf{x}^{*}, t\right)\right)^{T}$ is the depth-averaged velocity as function of horizontal coordinates $\mathbf{x}^{*}=\left(x^{*}, y^{*}\right)^{T}$ and time $t^{*}\left(\right.$ with $(\cdot)^{T}$ the transpose); and the free surface resides at $z^{*}=h^{*}+h_{b}^{*}$ with $h^{*}\left(\mathbf{x}^{*}, t\right)$ the total water depth and $h_{b}^{*}\left(\mathbf{x}^{*}\right)$ the elevation of the bottom topography above datum, both measured along the vertical coordinate, $z^{*}$, and aligned in the direction of the acceleration of gravity of magnitude $g^{*}$.

It is convenient to treat the SWE in non-dimensional form for computational reasons and to clarify the coupling of the flow to other physics phenomena, such as erosion and transport of sediments over an erodible bed, a process that introduces a sediment transport time scale much smaller than the flow time scale (see, e.g., Hall [17]). We therefore introduce the dimensionless variables

$$
\mathbf{x}=\mathbf{x}^{*} / l_{0}^{*}, \quad t=t^{*} / t_{0}^{*}, \quad h=h^{*} / h_{0}^{*}, \quad h_{b}=h_{b}^{*} / h_{0}^{*} \quad \text { and } \quad \mathbf{u}=\mathbf{u}^{*} / u_{0}^{*}
$$

where $l_{0}^{*}, t_{0}^{*}, h_{0}^{*}$ and $u_{0}^{*}$ are suitable, characteristic scales for the length of the fluid motion (usually a reference value of the channel width), time response, water depth and flow velocity, respectively. With (2.2), the system (2.1) reduces to a non-dimensional form, concisely written in index notation as

$$
\partial_{t} U_{i}+\partial_{j} F_{i j}(\mathbf{U})=S_{i} \text { in the flow domain } \Omega
$$

for $i=1,2,3$ and $\left(\partial_{x}, \partial_{y}\right)^{T}=\left(\partial_{1}, \partial_{2}\right)^{T}$ with $j=1,2$. Here, summation over repeated indices is understood. In $(2.3), \mathbf{U}=(h, h u, h v)^{T}$ is the vector of the 
conserved quantities, and

$$
F(\mathbf{U})=\left(\begin{array}{cc}
u h & v h \\
u^{2} h+\mathrm{F}^{-2} h^{2} / 2 & v u h \\
u v h & v^{2} h+\mathrm{F}^{-2} h^{2} / 2
\end{array}\right) \text { and } S=\left(\begin{array}{c}
0 \\
-\mathrm{F}^{-2} h \partial_{x} h_{b} \\
-\mathrm{F}^{-2} h \partial_{y} h_{b}
\end{array}\right)
$$

are flux and "source" terms, respectively. The Froude number $\mathrm{F}=u_{0}^{*} / \sqrt{g^{*} h_{0}^{*}}$ is the ratio between the flow velocity and surface gravity-wave speed. We emphasize that the variables $(h, h u, h v)^{T}$ are used to properly model bores and hydraulic jumps as discontinuities in a simple model of breaking waves, cf. the classical results in Lamb and Whitham [24, 41], summarized shortly. The numerical model presented later will thus include the physical energy dissipation across these jumps: this dissipation should be contrasted with any (much smaller) numerical dissipation in the numerical scheme. The system (2.3) and (2.4) is completed with initial conditions $\mathbf{U}(\mathbf{x}, 0)$, boundary conditions such as in- and outflow, and/or slip flow along solid walls. The latter are expressed as $\mathbf{U}=\mathbf{U}^{b}$ at the boundary $\partial \Omega$.

\subsection{Potential vorticity}

The vertical component of the vorticity, $\Lambda=\partial_{x} v-\partial_{y} u$, is conserved for smooth flows $([29])$,

$$
\partial_{t} \Lambda+\nabla \cdot(\mathbf{u} \Lambda)=0
$$

with spatial gradient operator $\boldsymbol{\nabla}=\left(\partial_{x}, \partial_{y}\right)^{T}$, which follows readily from $(2.1)$, or (2.3) and (2.4). By combining (2.5) with the mass equation, the $\mathrm{PV} \Pi=$ $\Lambda / h$ is found to be a materially conserved quantity ([29]),

$$
\partial_{t} \Pi+(\mathbf{u} \cdot \nabla) \Pi=0 .
$$

Bores and hydraulic jumps are discontinuities in the flow variables of the SWE, where energy is dissipated but mass and (local) momentum are conserved. In two dimensions these discontinuities occur along lines, and in one dimension at points, thus limiting multivalued solutions along lines and at points only. For a curvilinear bore we introduce a local coordinate system $(\eta, \tau)$ with $\eta$ in the bore direction and $\tau$ the direction along the bore, in the usual orientation. In the present configuration we take $h_{2}>h_{1}$ and $[h]=h_{1}-h_{2}$ with $h_{2}$ lying in the region where the bore has passed. The energy dissipation $E_{D}=B_{1}-B_{2}$, the difference between the Bernoulli function $B_{1}$ before and $B_{2}$ after passing of the bore with $B=(u-c)^{2} / 2+\mathrm{F}^{-2}\left(h+h_{b}\right)$ and $c$ the speed of the bore, is given by $([24,41])$

$$
E_{D}=\mathrm{F}^{-2}\left(h_{2}-h_{1}\right)^{3} /\left(4 h_{1} h_{2}\right) .
$$


Note that since $h_{2}>h_{1}$ dissipation $E_{D}$ is indeed positive such that energy is lost in the bore. Pratt [34] and Peregrine [30] show then that, in the presence of such discontinuities, the newly obtained $\mathrm{PV}, \Pi_{2}$, is

$$
\Pi_{2}-\Pi_{1}=-\sqrt{\frac{2}{\mathrm{~F}^{-2} h_{1} h_{2}\left(h_{1}+h_{2}\right)}} \frac{\partial E_{D}}{\partial \tau} .
$$

Bühler [10] shows that only PV anomalies can be generated by bores, such that the total PV remains the same in the absence of other sources or sinks of PV than the bores and hydraulic jumps.

\section{Space Discontinuous Galerkin Method}

\subsection{Space elements and tessellation}

The flow domain $\Omega \in \mathbb{R}^{2}$ is a bounded area which in turn is partitioned into $N_{e l}$ elements $K_{\mathrm{k}}$. It has a fixed boundary $\partial \Omega \in \mathbb{R}$. The tessellation of the domain $\Omega$ is defined as

$$
\mathcal{T}_{h}=\left\{K_{\mathrm{k}} \mid \bigcup_{\mathrm{k}=1}^{N_{e l}} \bar{K}_{\mathrm{k}}=\bar{\Omega}_{\mathrm{h}} \text { and } K_{\mathrm{k}} \cap K_{\mathrm{k}^{\prime}}=0 \text { if } \mathrm{k} \neq \mathrm{k}^{\prime}, 1 \leq \mathrm{k}, \mathrm{k}^{\prime} \leq N_{e l}\right\}
$$

such that the computational domain $\Omega_{\mathrm{h}} \rightarrow \Omega$ as $\mathrm{h} \rightarrow 0$, with $\mathrm{h}$ the magnitude of the smallest radius of the largest circle completely containing element $K_{\mathrm{k}} \in$ $\mathcal{T}_{h}$, and $\bar{K}_{\mathrm{k}}$ the closure of $K_{\mathrm{k}}$ (and likewise for $\bar{\Omega}$ ). Each element $K_{\mathrm{k}}$ can be transformed into a reference element $\hat{K}$ with the mapping

$$
F_{K}: \hat{K} \mapsto K_{\mathrm{k}}: \bar{\xi} \mapsto \mathbf{x}:=\sum_{j} \mathbf{x}_{j} \chi_{j}(\bar{\xi})
$$

where $\bar{\xi}=\left(\xi_{1}, \xi_{2}\right)$ are the reference coordinates, $\mathbf{x}_{j}$ are the coordinates of the local nodes $j=1, \ldots, N_{l, \mathrm{k}}$, and $\chi_{j}(\bar{\xi})$ the standard shape functions used in finite elements. For quadrilateral elements $N_{l, \mathrm{k}}=4$ and for triangular elements $N_{l, \mathrm{k}}=3$. In general, the element boundary $\partial K_{\mathrm{k}}$ is connected through faces $\mathcal{S}$ either to its neighboring elements or to the boundary of the domain. The set of all faces in $\Omega \cup \partial \Omega$ is denoted by $\Gamma$. The set of all interior faces in $\Omega$ is denoted by $\Gamma_{\text {int }}$ and the set of all boundary faces on $\partial \Omega$ is denoted by $\Gamma_{b o u}$. 


\subsection{Function spaces}

The finite element broken space is defined as

$$
\mathcal{V}_{\mathrm{h}}^{d}:=\left\{\mathbf{V}_{\mathrm{h}}\left|\mathbf{V}_{\mathrm{h}}\right|_{K} \in\left(P^{1}(K)\right)^{d}\right\}
$$

where $P^{1}$ is the space of linear polynomials, $d=\operatorname{dim}\left(\mathbf{V}_{\mathrm{h}}\right)$ and $\mathbf{V}_{\mathrm{h}}$ the polynomial approximation defined as $\mathbf{V}_{\mathbf{h}}:=\sum_{m} \hat{\mathbf{V}}_{m}(t) \psi_{m}(\mathbf{x})$ with $\hat{\mathbf{V}}_{m}$ the expansion coefficients and $\psi_{m}$ the polynomial basis functions. In the reference element $\hat{K}$, the set of polynomials is defined as

$$
\left\{\hat{\phi}_{0}, \hat{\phi}_{1}, \hat{\phi}_{2}, \hat{\phi}_{3}\right\}:=\left\{1, \xi_{1}, \xi_{2}, \xi_{1} \xi_{2}\right\}
$$

where the $\hat{\phi}_{m}(\bar{\xi})$ 's for $m=0,1,2,3$ are the basis functions on a quadrilateral element. On triangular elements, we use $\hat{\phi}_{m}(\bar{\xi})$ for $m=0,1,2$. If the topography $h_{b}(\mathbf{x})$ is discretized continuously, the extra function $\hat{\phi}_{3}$ is required on quadrilateral elements. It then enforces rest flow to be preserved numerically, see section 4.3. The local basis functions in $\hat{K}$ can be related to the basis functions in $K_{\mathrm{k}}$ by $\hat{\phi}_{m}(\bar{\xi})=\hat{\phi}_{m}\left(F_{K}^{-1}(\bar{\xi})\right)=\phi_{m}(\mathbf{x})$. We split the approximations of the conserved quantities in the space element $K_{\mathrm{k}}$ into mean and fluctuating parts. The basis functions are then redefined as

$$
\psi_{m}(\mathbf{x}, t)= \begin{cases}1 & \text { if } m=0 \\ \phi_{m}(\mathbf{x})-c_{m} & \text { otherwise }\end{cases}
$$

with $c_{m}=\frac{1}{\left|K_{\mathrm{k}}\right|} \int_{K_{\mathrm{k}}} \phi_{m}(\mathbf{x}) \mathrm{d} \mathbf{x}$. Here, $\left|K_{\mathrm{k}}\right|=\int_{K_{\mathrm{k}}} d K$ is the area of the element $K_{\mathrm{k}}$. If $\mathbf{x}$ is a point on a face $\mathcal{S}$ in $\Gamma$ and $\mathbf{n}_{K}$ is the outward unit normal vector of the boundary $\partial K_{\mathrm{k}}$, then the trace of the function $\mathbf{V}_{\mathrm{h}}$ on the element interior boundary $\partial K_{\mathrm{k}}$, relative to $K_{\mathrm{k}}$, is defined as $\left.\mathbf{V}_{\mathrm{h}}(\mathbf{x})\right|_{\partial K_{\mathrm{k}}}=\mathbf{V}^{-}:=\lim _{\epsilon \uparrow 0} \mathbf{V}_{\mathrm{h}}(\mathbf{x}-$ $\left.\epsilon \mathbf{n}_{K}\right)$. The traces of the functions on each face relative to the neighboring element are, in general, discontinuous since $\mathbf{V}_{\mathrm{h}}(\mathbf{x}) \in \mathcal{V}_{\mathrm{h}}^{d}$.

\subsection{Weak formulation}

The space discontinuous Galerkin weak formulation is obtained by multiplying (2.3) by a test function $\mathbf{W}_{\mathrm{h}}$ in the finite element space $\mathcal{V}_{\mathrm{h}}^{d}$, integrating over the space element $K_{\mathrm{k}}$, and replacing the exact solution $\mathbf{U}$ by its approximation $\mathbf{U}_{\mathrm{h}} \in \mathcal{V}_{\mathrm{h}}^{d}$

$$
\int_{K_{\mathrm{k}}} W_{\mathrm{h} i} \partial_{t} U_{\mathrm{h} i} \mathrm{~d} K+\int_{K_{\mathrm{k}}} W_{\mathrm{h} i} \partial_{j} F_{i j}\left(\mathbf{U}_{\mathrm{h}}\right) \mathrm{d} K-\int_{K_{\mathrm{k}}} W_{\mathrm{h} i} S_{i} \mathrm{~d} K=0 .
$$


Applying Gauss's theorem to the flux term of (3.6) and after summing over all elements, the weak formulation is: find $a \mathbf{U}_{\mathrm{h}} \in \mathcal{V}_{\mathrm{h}}^{d}$ such that for all $\mathbf{W}_{\mathrm{h}} \in \mathcal{V}_{\mathrm{h}}^{d}$

$$
\begin{gathered}
\sum_{K_{\mathrm{k}}}\left\{\int_{K_{\mathrm{k}}} W_{\mathrm{h} i} \partial_{t} U_{\mathrm{h} i} \mathrm{~d} K+\int_{\partial K_{\mathrm{k}}} n_{K j} W_{\mathrm{h} i}^{-} F_{i j}{ }^{-} \mathrm{d} \Gamma-\right. \\
\left.\int_{K_{\mathrm{k}}} \partial_{j} W_{\mathrm{h} i} F_{i j}\left(\mathbf{U}_{\mathrm{h}}\right) \mathrm{d} K-\int_{K_{\mathrm{k}}} W_{\mathrm{h} i} S_{i} \mathrm{~d} K\right\}=0
\end{gathered}
$$

is satisfied, where $W_{\mathrm{h} i}^{-}$and $U_{\mathrm{h} i}^{-}$are the traces of $W_{\mathrm{h} i}$ and $U_{\mathrm{h} i}$ respectively; $F_{i j}^{-}=$ $F_{i j}\left(\mathbf{U}_{\mathrm{h}}^{-}\right)$; and $\mathrm{d} \Gamma$ is an infinitesimal boundary segment. The communication of mass and momentum between neighboring elements occurs through the element faces.

In the weak formulation (3.6), there is thus an ambiguity because $U_{\mathrm{h} i}$ is discontinuous, implying that the flux $F$ at a face is discontinuous. Considering the integrals over the element boundaries with a counterclockwise ordering such that $n_{K j}^{l}, F_{i j}^{l}$, and $W_{\mathrm{h} i}^{l}$ are the outward normal, the flux, and the test function for a left element, and $n_{K j}^{r}, F_{i j}^{r}$, and $W_{\mathrm{h} i}^{r}$ the corresponding quantities for the right element, we find

$$
\begin{aligned}
& \sum_{K_{\mathrm{k}}} \int_{\partial K_{\mathrm{k}}} n_{K j} W_{\mathrm{hi}}^{-} F_{i j}{ }^{-} \mathrm{d} \Gamma=\sum_{\mathcal{S}} \int_{\mathcal{S}}\left(n_{K j}^{l} W_{\mathrm{h} i}^{l} F_{i j}^{l}+n_{K j}^{r} W_{\mathrm{h} i}^{r} F_{i j}^{r}\right) \mathrm{d} \Gamma= \\
& \sum_{\mathcal{S}} \int_{\mathcal{S}}\left(\alpha \mathcal{F}_{i j}^{l}+\beta F_{i j}^{r}\right)\left(n_{K j}^{l} W_{\mathrm{hi}}^{l}+n_{K j}^{r} W_{\mathrm{h} i}^{r}\right)+\left(n_{K j}^{l} F_{i j}^{l}+n_{K j}^{r} F_{i j}^{r}\right)\left(\alpha W_{\mathrm{h} i}^{l}+\beta W_{\mathrm{h} i}^{r}\right) \mathrm{d} \Gamma
\end{aligned}
$$

with $\alpha+\beta=1$ and $\alpha, \beta \geq 0$. If the flux is taken to be conservative such that

$$
\int_{\mathcal{S}} n_{K j}^{l} W_{\mathrm{h} i}^{l} F_{i j}^{l} \mathrm{~d} \Gamma=-\int_{\mathcal{S}} n_{K j}^{r} W_{\mathrm{h} i}^{r} F_{i j}^{r} \mathrm{~d} \Gamma
$$

and $n_{K j}^{l}=-n_{K j}^{r}$ at a face, then

$$
\sum_{K_{\mathrm{k}}} \int_{\partial K_{\mathrm{k}}} n_{K j} W_{\mathrm{hi}}^{-} F_{i j}^{-} \mathrm{d} \Gamma=\sum_{\mathcal{S}} \int_{\mathcal{S}} n_{K j}^{l}\left(W_{\mathrm{hi}}^{l}-W_{\mathrm{h} i}^{r}\right)\left(\alpha F_{i j}^{l}+\beta F_{i j}^{r}\right) \mathrm{d} \Gamma .
$$

The flux $\left(\alpha F_{i j}^{l}+\beta F_{i j}^{r}\right)$ in (3.9) is replaced by a numerical flux $\tilde{F}_{i j}\left(\mathbf{U}^{l}, \mathbf{U}^{r}, \mathbf{n}_{K}\right)$, which depends on the discontinuous trace values $\mathbf{U}^{l}$ and $\mathbf{U}^{r}$ directly adjacent to each interface. The boundary condition $\mathbf{U}^{r}=\mathbf{U}^{b}$ is applied on faces belonging to a boundary $\mathcal{S} \in \Gamma_{\text {bou }}$. Finally, the weak formulation for the space DG method for each space element now becomes: find $a \mathbf{U}_{\mathrm{h}} \in \mathcal{V}_{\mathrm{h}}^{d}$ such that for all $\mathbf{W}_{\mathrm{h}} \in \mathcal{V}_{\mathrm{h}}^{d}$

$$
\begin{array}{r}
\sum_{\mathcal{S}}\left\{\int_{\mathcal{S}} n_{K j} \tilde{F}_{i j}\left(\mathbf{U}^{l}, \mathbf{U}^{r}, \mathbf{n}_{K}\right)\left(W_{\mathrm{h} i}^{l}-W_{\mathrm{h} i}^{r}\right) \mathrm{d} \Gamma\right\}+ \\
\sum_{K}\left\{\int_{K_{\mathrm{k}}} W_{\mathrm{h} i} \partial_{t} U_{\mathrm{h} i} \mathrm{~d} K-\int_{K_{\mathrm{k}}} \partial_{j} W_{\mathrm{h} i} F_{i j}\left(\mathbf{U}_{\mathrm{h}}\right) \mathrm{d} K-\int_{K_{\mathrm{k}}} W_{\mathrm{h} i} S_{i} \mathrm{~d} K\right\}=0
\end{array}
$$


is satisfied. This form is also used in the numerical implementation. Numerically, the element and face integrals are approximated using Gauss quadrature.

\subsection{Numerical flux}

In this work, the HLLC numerical flux of Toro et al. [37] and the kinetic numerical flux are used. The HLLC flux is an accurate and efficient approximation to a numerical flux based on a one-dimensional Riemann problem in the direction normal to each face at the relevant quadrature points. It is a modification of the HLL [18] scheme that takes into account the influence of contact waves [5, 37]. On the other hand, the kinetic numerical flux is based on the theory of kinetic schemes for gas dynamics and links Vlasov equations with hydrodynamic equations [33]. The main difference of the kinetic flux with respect to the HLLC flux is the incorporation of the topographic term in the derivation of the approach. Their formulations are given next.

\subsubsection{HLLC numerical flux}

If we suppose that the element face $\mathcal{S}$, whose unit normal vector $\mathbf{n}_{K}=$ $\left(n_{K x}, n_{K y}\right)^{T}$ is oriented is such a way that $\partial_{\hat{x}}=\mathbf{n}_{K} \cdot \boldsymbol{\nabla}$ defines the derivative normal to the face $\mathcal{S}$, then the normal flux is determined by considering the SWE (i.e., (2.3 and (2.4) without source terms) along $\mathbf{n}_{K}$, and the following one-dimensional Riemann problem can be distilled from the SWE

$$
\partial_{t} \mathbf{U}+\partial_{\hat{x}} \hat{\mathbf{F}}(\mathbf{U})=0
$$

with initial constant states $\mathbf{U}^{l}$ and $\mathbf{U}^{r}$ left and right of each face, $\hat{\mathbf{F}}(\mathbf{U})=$ $q \mathbf{U}+\hat{\mathbf{P}}, q=\mathbf{n}_{K} \cdot \mathbf{u}$ the normal velocity, $\mathbf{u}=(u, v)^{T}, \hat{\mathbf{P}}=\left(0, n_{K x} P, n_{K y} P\right)^{T}$ the normal pressure, and $P=\mathrm{F}^{-2} h^{2} / 2$ the effective pressure. The HLLC approximation to the normal flux vector $\hat{\mathbf{F}}(\mathbf{U})$ at the cell boundary is proposed next and is based on this Riemann problem.

The HLLC approach for the case of a wet bed assumes that there are four states from left to right, that is $\mathbf{U}^{l}, \mathbf{U}^{* l}, \mathbf{U}^{* r}, \mathbf{U}^{r}$, separated by left $(l)$, right $(r)$, and middle $(m)$ transition lines defined by wave speeds $s_{l}, s_{r}, s_{m}$, respectively

$$
\mathbf{U}=\left\{\begin{array}{cc}
\mathbf{U}^{l} & \hat{x} / t<s_{l} \\
\mathbf{U}^{* l} & s_{l}<\hat{x} / t<s_{m} \\
\mathbf{U}^{* r} & s_{m}<\hat{x} / t<s_{r} \\
\mathbf{U}^{r} & \hat{x} / t>s_{r}
\end{array},\right.
$$


where $\mathbf{U}^{* l, r}$ are the averaged intermediate states and $\hat{x}=0$ is chosen to coincide with the relevant face. After integration of (3.11) over two control volumes, see $[5,37], \hat{\mathbf{F}}$ is computed as the average of the contribution on either side of the face. Using Gauss' theorem in space and time, the HLLC flux is

$$
\begin{aligned}
\hat{\mathbf{F}}\left(\mathbf{U}^{l}, \mathbf{U}^{r}\right)= & \frac{1}{2}\left\{\hat{\mathbf{F}}^{l}+\hat{\mathbf{F}}^{r}-\left(\left|s_{l}\right|-\left|s_{m}\right|\right) \mathbf{U}^{* l}\right. \\
& \left.+\left(\left|s_{r}\right|-\left|s_{m}\right|\right) \mathbf{U}^{* r}+\left|s_{l}\right| \mathbf{U}^{l}-\left|s_{r}\right| \mathbf{U}^{r}\right\}
\end{aligned}
$$

where $\hat{\mathbf{F}}^{l, r}=\hat{\mathbf{F}}\left(\mathbf{U}^{l, r}\right)$. From the HLL approximation, an expression for the intermediate state $\mathbf{U}^{*}$ is obtained [5]

$$
\mathbf{U}^{*}=\left(s_{r} \mathbf{U}^{r}-s_{l} \mathbf{U}^{l}-\left(\hat{\mathbf{F}}^{r}-\hat{\mathbf{F}}^{l}\right)\right) /\left(s_{r}-s_{l}\right) .
$$

An expression for speed $s_{m}$ can be found [5] assuming that $s_{m}=q_{l}^{*}=q_{r}^{*}=q^{*}$, where $q^{*}$ is the average velocity between the left and right waves. From (3.14) and the latter expression, the following wave speed $s_{m}$ is obtained

$$
s_{m}=q^{*}=\mathbf{n}_{K} \cdot \mathbf{u}^{*}=\frac{P_{l}-P_{r}+h_{r} q_{r}\left(s_{r}-q_{r}\right)-h_{l} q_{l}\left(s_{l}-q_{l}\right)}{h_{r}\left(s_{r}-q_{r}\right)-h_{l}\left(s_{l}-q_{l}\right)} .
$$

By using the Rankine-Hugoniot relations for (3.11), the intermediate states $U^{* l}$ and $U^{* r}$ are found to be

$$
\left(s_{l, r}-s_{m}\right) U_{i}^{* l, * r}=\left(s_{l, r}-q_{l, r}\right) U_{i}^{l, r}+\left(\hat{P}_{i}^{* l, * r}-\hat{P}_{i}^{l, r}\right),
$$

where the average intermediate normal pressure $P_{i}^{* l, * r}=\left(0, n_{K x} P_{l, r}^{*}, n_{K y} P_{l, r}^{*}\right)^{T}$. After some algebraic manipulations of (3.16), we find $P^{*}=P_{l, r}^{*}=h_{l, r}\left(q_{l, r}\right.$ $\left.s_{l, r}\right)\left(q_{l, r}-s_{m}\right)+P_{l, r}$.

Finally, the wave speeds are estimated based on the left and right moving rarefaction wave speeds $s_{l}=\min \left(q_{l}-a_{l}, q_{r}-a_{r}\right)$ and $s_{r}=\max \left(q_{l}+a_{l}, q_{r}+a_{r}\right)$, respectively, with $a^{2}=\partial P / \partial h$. When $s_{l}>0$ the flux simplifies to $\hat{\mathbf{F}}^{l}$, and when $s_{r}<0$ to $\hat{\mathbf{F}}^{r}$, that is, the classic upwind cases. We refer to [5] for more information.

\subsubsection{Kinetic numerical flux}

Consider the collisionless kinetic or Vlasov equation $[28,7]$

$$
\partial_{t} \mathrm{D}+\boldsymbol{\nabla} \cdot(\bar{\zeta} \mathrm{D})-\nabla_{\zeta} \cdot\left[\left(\mathrm{F}^{-2} \nabla h_{b}\right) \mathrm{D}\right]=0
$$

with distribution function $\mathrm{D}=\mathrm{D}(\mathbf{x}, \bar{\zeta}, t)$ a function of horizontal coordinates $\mathbf{x}$, velocity coordinates $\bar{\zeta}=\left(\zeta_{1}, \zeta_{2}\right)^{T}$ and time; potential $h_{b}(\mathbf{x}) / \mathrm{F}^{2}$; and a velocity 
gradient operator $\boldsymbol{\nabla}_{\zeta}$. Note that the Vlasov equation is a conservation law linear in D.

Consider a function $\chi: \mathcal{R}^{2} \rightarrow \mathcal{R}^{+}$with the following symmetry properties

$$
\chi(\cdot) \geq 0, \quad \chi(\bar{\omega})=\chi(-\bar{\omega}), \quad \int_{\mathcal{R}} \chi(\bar{\omega}) \mathrm{d} \bar{\omega}=1, \quad \int_{\mathcal{R}} \omega_{i}^{2} \chi(\bar{\omega}) \mathrm{d} \bar{\omega}=1
$$

with $\bar{\omega}=\left(\omega_{1}, \omega_{2}\right), \mathrm{d} \bar{\omega}=\mathrm{d} \omega_{1} \mathrm{~d} \omega_{2}$, and $i=1,2$. Hence, $\int_{\mathcal{R}} \omega_{i} \chi(\bar{\omega}) \mathrm{d} \bar{\omega}=0$. The distribution function $D$ is restricted to be in the equilibrium state

$$
\mathrm{D}=K(h) \chi((\bar{\xi}-\mathbf{u}) / G(h))
$$

with (Eulerian) fluid velocity $\mathbf{u}=\mathbf{u}(\mathbf{x}, t)$ and pseudo-density $h=h(\mathbf{x}, t)$. We define the functions $K(h)$ and $G(h)$ such that

$$
h=K G^{2} \quad \text { and } \quad P(h)=K G^{4}=h^{2} /(2 \mathrm{~F})
$$

with $P(h)$ the effective pressure, a smooth, increasing function of $h$.

Proposition 3.1 The two-dimensional shallow water equations (2.3) and (2.4) follow from the kinetic equation of motion (3.17) when the distribution function is constrained to (3.19) and (3.20) for a function $\chi(\cdot)$ in (3.19) with symmetry properties (3.18).

Proof: see Appendix A.

Note that (3.17) can also be written in the concise form (2.3) by taking $U=$ $\mathrm{D}(\mathbf{x}, \bar{\zeta}, t), F=\bar{\zeta} \mathrm{D}$, and $S=\nabla_{\zeta} \cdot\left[\left(\mathrm{F}^{-2} \nabla h_{b}\right) \mathrm{D}\right]$.

\subsubsection{Weak formulation from kinetic formulation}

A discretization of the equations of motion (2.3) and (2.4) for the $P(h)$-fluid can be formulated from a discretization of the kinetic equation (3.17). In this way, one directly obtains a numerical flux. In contrast to the higherorder discontinuous finite-element discretization proposed here, Perthame [32] and Perthame and Simeoni [33] considered a kinetic flux formulation for a first-order finite-volume discretization of the compressible Euler equations and SWE, respectively.

The weak formulation for the kinetic equations of motion (3.17) is

$$
\begin{array}{r}
\sum_{K_{\mathrm{k}}} \int_{K_{\mathrm{k}}} w_{\mathrm{h}} \partial_{t} \mathrm{D} \mathrm{d} K+\int_{\partial K_{\mathrm{k}}}\left(\hat{n}_{\mathrm{k}}^{-} \cdot \bar{\zeta}\right) w_{\mathrm{h}}^{-} \tilde{\mathrm{D}}_{\mathrm{h}} \mathrm{d} \Gamma- \\
\int_{K_{\mathrm{k}}} \mathrm{D}_{\mathrm{h}} \nabla w_{\mathrm{h}} \cdot \bar{\zeta} \mathrm{d} K-\int_{K_{\mathrm{k}}} w_{\mathrm{h}}\left(f \bar{\zeta}^{\perp}+\mathrm{F}^{-2} \nabla h_{b}\right) \cdot \nabla_{\zeta} \mathrm{D}_{\mathrm{h}} \mathrm{d} K=0,
\end{array}
$$


where we used the upwind flux

$$
\tilde{\mathrm{D}}=\mathrm{D}_{\mathrm{k}}^{-} \Theta\left(\hat{n}_{\mathrm{k}}^{-} \cdot \bar{\zeta}\right)+\mathrm{D}_{\mathrm{k}}^{+} \Theta\left(-\hat{n}_{\mathrm{k}}^{-} \cdot \bar{\zeta}\right)
$$

with Heaviside function $\Theta(\cdot)$ (i.e. $\Theta(a)=0$ for $a<0$ and $\Theta(a)=1$ for $a \geq 0$ ), and traces $D_{k}^{-}$and $D_{k}^{+}$of $D$ in- and outside the element along the faces of the element $K_{\mathrm{k}}$. The test function $w_{\mathrm{h}}$ is a scalar function since D is a scalar function, in contrast to the test functions introduced in the weak formulation (3.6) for the SWE. The DG numerical approximation in space, $\mathbf{x}$, of $D$ is $D_{h}$.

Multiplying $(3.21)$ by $(1, \bar{\zeta})^{T}$, integrating over $\bar{\zeta}$, and applying the same operations as in the continuous case, yields a numerical discretization of the SWE

$$
\begin{array}{r}
\int_{K_{\mathrm{k}}} w_{\mathrm{h}} \frac{\mathrm{d} U_{\mathrm{h} i}}{\mathrm{~d} t} \mathrm{~d} K+\int_{\partial K_{\mathrm{k}}} w_{\mathrm{h}}^{-} \tilde{F}_{i j}^{k i n} \mathrm{~d} \Gamma-\int_{K_{\mathrm{k}}} \partial_{j} w_{\mathrm{h}} F_{i j}\left(\mathbf{U}_{\mathrm{h}}\right) \mathrm{d} K+ \\
\int_{K_{\mathrm{k}}} w_{\mathrm{h}} h_{\mathrm{h}}\left(0, f \mathbf{u}^{\perp}+\mathrm{F}^{-2} \nabla h_{b}\right)_{i}^{T} \mathrm{~d} K=0
\end{array}
$$

with the numerical kinetic flux vector

$$
\begin{aligned}
\tilde{F}^{k i n} & =\tilde{F}^{\text {out }}\left(\mathbf{U}^{l}\right)+\tilde{F}^{\text {in }}\left(\mathbf{U}^{r}\right) \\
\tilde{F}^{\text {out }}\left(\mathbf{U}^{l}\right) & =\int \mathrm{D}^{l}\left(\hat{n}_{\mathrm{k}}^{-} \cdot \bar{\zeta}\right) \Theta\left(\hat{n}_{\mathrm{k}}^{-} \cdot \bar{\zeta}\right)(1, \bar{\zeta})^{T} \mathrm{~d} \bar{\zeta} \\
\tilde{F}^{\text {in }}\left(\mathbf{U}^{r}\right) & =\int \mathrm{D}^{r}\left(\hat{n}_{\mathrm{k}}^{-} \cdot \bar{\zeta}\right) \Theta\left(-\hat{n}_{\mathrm{k}}^{-} \cdot \bar{\zeta}\right)(1, \bar{\zeta})^{T} \mathrm{~d} \bar{\zeta}
\end{aligned}
$$

For $S=\left(0, \mathrm{~F}^{-2} \nabla h_{b}\right)^{T}$, the difference between the weak formulation (3.10) with the HLLC numerical flux and (3.23) lies in the definition of the numerical flux and the test functions $W_{\mathrm{h}}$ and $w_{\mathrm{h}}$ Further information about the implementation of the kinetic flux is found in [36].

\subsection{Stabilization operator and discontinuity detector for $D G$}

Higher-order numerical schemes produce spurious oscillations around discontinuities that can lead to numerical instabilities and to unbounded results in finite time. As pointed out in [26], only schemes that are first-order accurate are able to produce monotonic solutions when discontinuities are present. Such schemes produce too much numerical dissipation, which severely deteriorates the solution quality. Several flux limiting strategies have been developed to cope with spurious oscillations [25]. The approach presented here uses the jump in the polynomial representation of the solution at the element faces in the discontinuous Galerkin discretization, and is based on the approach followed by [38] for a space-time discontinuous Galerkin method. We add the 
following stabilizing operator to the weak formulation per space element $K_{\mathrm{k}}$ :

$$
\int_{K_{\mathrm{k}}} \partial_{l} W_{\mathrm{h} i} \mathcal{D}_{\mathrm{k}, l j}\left(\mathbf{U}_{\mathrm{h}}^{l}, \mathbf{U}_{\mathrm{h}}^{r}\right) \partial_{j} U_{\mathrm{h} i} d K
$$

where $\mathbf{U}_{\mathrm{h}}^{l, r}$ is the discontinuous Galerkin solution in neighboring elements and $\mathcal{D}_{\mathrm{k}, l j}\left(\mathbf{U}_{\mathrm{h}}^{l}, \mathbf{U}_{\mathrm{h}}^{r}\right)$ is the dissipation matrix. Instead of flux limiting after each time step, we include it in the weak formulation, which is advantageous for steady state calculations. Since it is desirable to obtain solutions with neither excessive amount of dissipation nor spurious oscillations, a discontinuity detector proposed by Krivodonova et al. [23] is implemented for the SWE, defined as

$$
\mathcal{I}_{\mathrm{k}}=\frac{\sum_{\mathcal{S} \in \partial K_{\mathrm{k}}} \int_{\mathcal{S}}\left|h_{\mathrm{h}}^{r}-h_{\mathrm{h}}^{l}\right| d \mathcal{S}}{\mathbf{s}_{K}^{(p+1) / 2}\left|\partial K_{\mathrm{k}}\right| \max \left\|h_{\mathrm{h}}\right\|},
$$

where $\left|\partial K_{\mathrm{k}}\right|$ is the length of the element boundary, $\mathrm{s}_{K}$ is the cell measure defined as the radius of the largest circumscribed circle in the element $K_{\mathrm{k}}, p$ is the order of the polynomial used, $h_{\mathrm{h}}^{l, r}$ is the approximate water depth in adjacent elements, and $\max \|\cdot\|$ the maximum norm based on local solution maxima at Gauss integration points in the element $K_{\mathrm{k}}$. The solution is smooth if $\mathcal{I}_{\mathrm{k}}>1$ and the solution is discontinuous if $\mathcal{I}_{\mathrm{k}}<1$ [23].

Combining the stabilization operator (3.27) with the discontinuity detector (3.28), and upon introducing the Heaviside step function $\Theta\left(\mathcal{I}_{\mathbf{k}}-1\right)$, the weak formulation reduces to: find a $\mathbf{U}_{\mathrm{h}} \in \mathcal{V}_{\mathrm{h}}^{d}$ such that for all $\mathbf{W}_{\mathrm{h}} \in \mathcal{V}_{\mathrm{h}}^{d}$

$$
\begin{array}{r}
\left.\sum_{\mathcal{S}}\left\{\int_{\mathcal{S}} n_{K j} \tilde{F}_{i j}\left(\mathbf{U}^{l}, \mathbf{U}^{r}, \mathbf{n}_{K}\right)\right)\left(W_{\mathrm{h} i}^{l}-W_{\mathrm{h} i}^{r}\right) \mathrm{d} \Gamma\right\}+ \\
\sum_{K}\left\{\int_{K_{\mathrm{k}}} W_{\mathrm{h} i} \partial_{t} U_{\mathrm{h} i} \mathrm{~d} K-\int_{K_{\mathrm{k}}} \partial_{j} W_{\mathrm{h} i} F_{i j}\left(\mathbf{U}_{\mathrm{h}}\right) \mathrm{d} K-\int_{K_{\mathrm{k}}} W_{\mathrm{h} i} S_{i}-\right. \\
\left.\Theta\left(\mathcal{I}_{\mathrm{k}}-1\right) \int_{K_{\mathrm{k}}} \partial_{l} W_{\mathrm{h} i} \mathcal{D}_{\mathrm{k}, l j}\left(\mathbf{U}_{\mathrm{h}}^{l}, \mathbf{U}_{\mathrm{h}}^{r}\right) \partial_{j} U_{\mathrm{h} i} d K\right\}=0
\end{array}
$$

is satisfied.

\subsection{Stabilization matrix}

An artificial viscosity matrix is introduced such that the stabilization operator operates independently in all computational coordinate directions using the relation

$$
\mathcal{D}_{\mathrm{k}}\left(\mathbf{U}_{\mathrm{h}}^{l}, \mathbf{U}_{\mathrm{h}}^{r}\right)=\mathcal{R}^{T} \tilde{\mathcal{D}}_{\mathrm{k}}\left(\mathbf{U}_{\mathrm{h}}^{l}, \mathbf{U}_{\mathrm{h}}^{r}\right) \mathcal{R}
$$

where the matrix $\mathcal{R}$ is defined as $\mathcal{R}=2 \mathcal{H}^{-1} \boldsymbol{\nabla} F_{K}$ and $\tilde{\mathcal{D}}_{\mathrm{k}}\left(\mathbf{U}_{\mathrm{h}}^{l}, \mathbf{U}_{\mathrm{h}}^{r}\right)$ is the stabilization matrix in reference coordinates. The diagonal matrix $\mathcal{H}$ is introduced to ensure that both $\mathcal{D}_{\mathrm{k}}\left(\mathbf{U}_{\mathrm{h}}^{l}, \mathbf{U}_{\mathrm{h}}^{r}\right)$ and $\tilde{\mathcal{D}}_{\mathrm{k}}\left(\mathbf{U}_{\mathrm{h}}^{l}, \mathbf{U}_{\mathrm{h}}^{r}\right)$ have the same mesh 
dependence as function of $c_{i}$, defined as $\mathcal{H}=\operatorname{diag}\left(c_{1}, c_{2}\right)$, where the $c_{i}$ 's are the leading terms of the expansion of the mapping $F_{K}$ in the reference coordinates. For quadrilaterals $c_{1}=\left|\mathbf{x}_{1}+\mathbf{x}_{2}-\mathbf{x}_{0}-\mathbf{x}_{3}\right| / 4$ and $c_{2}=\left|\mathbf{x}_{2}+\mathbf{x}_{3}-\mathbf{x}_{0}-\mathbf{x}_{1}\right| / 4$. For the evaluation of the coefficients $\tilde{\mathcal{D}}_{\mathrm{k}, q q}\left(\mathbf{U}_{\mathrm{h}}^{l}, \mathbf{U}_{\mathrm{h}}^{r}\right)$ of the stabilization matrix, the artificial viscosity model proposed by Jaffre et al. [21] is adopted here

$$
\begin{cases}\tilde{\mathcal{D}}_{\mathrm{k}, q q}\left(\mathbf{U}_{\mathrm{h}}^{l}, \mathbf{U}_{\mathrm{h}}^{r}\right)=C c_{K}^{2} R_{\mathrm{k}}\left(\mathbf{U}_{\mathrm{h}}^{l}, \mathbf{U}_{\mathrm{h}}^{r}\right) & \text { for } q=1,2 \\ 0, & \text { otherwise }\end{cases}
$$

with the residual $\left.R_{\mathrm{k}}\left(\mathbf{U}_{\mathrm{h}}^{l}, \mathbf{U}_{\mathrm{h}}^{r}\right)\right)=\sum_{\mathcal{S} \in \partial K_{\mathrm{k}}} \frac{1}{c_{K}} \max \left\|n_{K j}^{l}\left(F_{i j}^{l}-F_{i j}^{r}\right)\right\|$, where $c_{K}=\sqrt{c_{1}^{2}+c_{2}^{2}}$ the scaling factor, $\|\cdot\|$ is based on the maximum at the local Gauss integration points, and $C$ is a positive constant with values between the range $[0.01 ; 0.1]$.

\subsection{Time discretization}

The discretized weak formulation arises when the test functions $W_{\mathrm{h} i}$ are alternately chosen as the functions $\psi_{m}$. It consists of a system of ordinary differential equations. We use the explicit third order TVD Runge Kutta scheme of [35] to step forward in time. The associated time step constraint has the form $\Delta t=C F L \min _{K_{\mathrm{k}}}\left(\left|K_{\mathrm{k}}\right| / \max _{K_{\mathrm{k}}}|\mathbf{u} \pm \sqrt{h} / \mathrm{F}|\right)$ with the Courant-Friedrichs-Lewy number $C F L \leq 1$.

\section{Verification of the model}

The discontinuous Galerkin finite element method has been implemented on a quadrilateral mesh using the $\mathrm{C}++$ programming language. To verify numerical solutions against exact ones, regular as well as irregular meshes are used. The latter are constructed by a restricted, random movement of the internal nodes of a regular grid. The error in the $L_{2}$-norm,

$$
\left(\int_{\Omega_{\mathrm{h}}}\left(\mathbf{U}_{\mathrm{e}}-\mathbf{U}_{\mathrm{h}}\right)^{2} d \Omega\right)^{1 / 2}=\left(\sum_{K} \int_{K_{\mathrm{h}}}\left(\mathbf{U}_{\mathrm{e}}-\mathbf{U}_{\mathrm{h}}\right)^{2} d \Omega\right)^{1 / 2}
$$

is used in the verification tests with $\mathbf{U}_{\mathrm{e}}$ and $\mathbf{U}_{\mathrm{h}}$ the exact and computed solutions, respectively. These exact solutions are described in Appendix B. 
Table 1

\begin{tabular}{llllll}
\hline \multirow{2}{*}{$N \times N$} & $h$ & & & $h u$ & \\
\cline { 2 - 3 } \cline { 5 - 6 } & $L_{2}$ error & Order $p$ & & $L_{2}$ error & Order $p$ \\
\hline $20 \times 20$ & $1.049 \mathrm{e}-03$ & & & $5.003 \mathrm{e}-04$ & \\
$40 \times 40$ & $2.796 \mathrm{e}-04$ & 1.90 & & $1.300 \mathrm{e}-04$ & 1.94 \\
$80 \times 80$ & $7.329 \mathrm{e}-05$ & 1.93 & & $3.297 \mathrm{e}-05$ & 1.97 \\
$160 \times 160$ & $1.888 \mathrm{e}-05$ & 1.95 & & $8.291 \mathrm{e}-06$ & 1.99 \\
\hline
\end{tabular}

The $L_{2}$ error of $h$ and $h u$ and convergence rates for Burgers' solution of the SWE on non-uniform meshes are shown for the HLLC flux.

Table 2

\begin{tabular}{llllll}
\hline$N \times N$ & $h$ & & & $h u$ & \\
\cline { 2 - 3 } \cline { 5 - 6 } & $L_{2}$ error & Order $p$ & & $L_{2}$ error & Order $p$ \\
\hline $20 \times 20$ & $9.809 \mathrm{e}-04$ & & & $4.629 \mathrm{e}-04$ & \\
$40 \times 40$ & $2.556 \mathrm{e}-04$ & 1.94 & & $1.185 \mathrm{e}-04$ & 1.96 \\
$80 \times 80$ & $6.593 \mathrm{e}-05$ & 1.95 & & $2.973 \mathrm{e}-05$ & 1.99 \\
$160 \times 160$ & $1.727 \mathrm{e}-05$ & 1.93 & & $7.650 \mathrm{e}-06$ & 1.96 \\
\hline
\end{tabular}

The $L_{2}$ error for $h$ and $h u$ and convergence rates for Burgers' solution on nonuniform meshes are shown for the kinetic flux.

\section{1 "Burgers' solution"}

A solution is found of one characteristic of the one-dimensional SWE, symmetrized to vary only in the $x$-direction, while the other characteristic is fixed. The former characteristic is then reduced to Burgers' equation with use of the latter characteristic, and the resulting Burgers' equation is solved implicitly. These shallow water solutions are valid before the onset of breaking in Burgers' equation. Numerical simulations were carried out in a domain $\Omega=1 \times 1$ with initial condition $\sqrt{K-9 h(x, 0)^{2} \mathrm{~F}^{-2}}=0.4 \sin (2 \pi x)$, and $u=K-2 \mathrm{~F}^{-1} \sqrt{h}$, $\mathrm{F}=1$ and $K=2$. At the zonal boundaries (at $x=0$ and $x=1$ ), periodic boundary conditions were imposed or the exact solutions. Slip flow was imposed at the solid meridional boundaries $(y=0$ and $y=1)$. At time $t=t_{b}=1 /(0.8 \pi) \approx 0.4$ the initially smooth solution develops a discontinuity. The $L_{2}$-norm error and the order of accuracy on non-uniform grids at time $t=0.2$ are given for mass $h$ and momentum $h u$ in Tables 1 and 2 for the HLLC and kinetic numerical fluxes on the same meshes, respectively. It shows that the numerical scheme is second order accurate. Note that the two-dimensional code is tested on these non-uniform grids. 


\subsection{Dam-break problem}

To demonstrate the shock-capturing ability of the numerical scheme, a Riemann problem consisting of an idealized dam break flow for the one-dimensional SWE is considered in a domain $\Omega=1 \times 1$ with extrapolating boundary conditions and initial condition: $h(x, 0)=1$ for $x \leq x_{0}$ and $h(x, 0)=0.10$ for $x>x_{0}$ and rest flow $\mathbf{u}(x, 0)=0$. At $t=0$, the dam is removed and the solution develops into a rarefaction wave propagating upstream and a hydraulic jump propagating downstream. Numerical and exact profiles of the water depth $h$ are shown in Figure (1a) from time $t=0$ to time $t=4$ for the kinetic numerical flux (a similar result holds for the HLLC flux). Krivodonova's discontinuity detector predicts the bore region well, see Figure 1b), and the agreement between the numerical and exact solution is good.

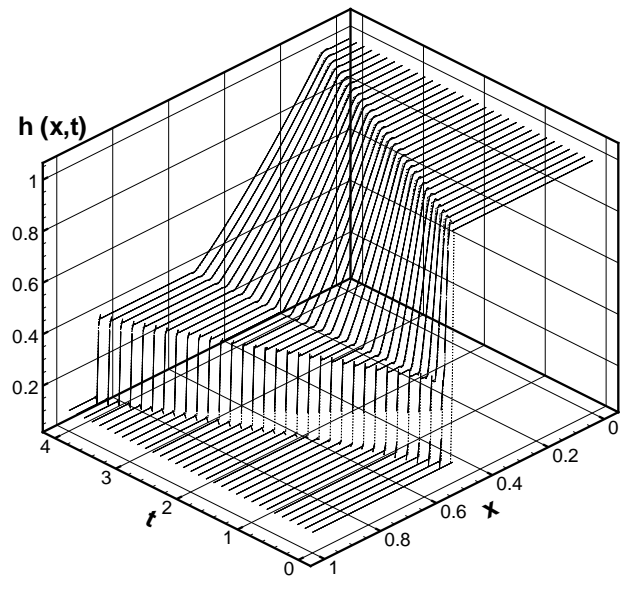

(a)

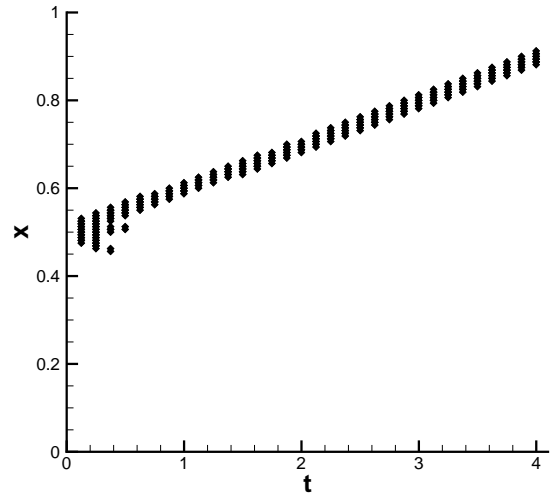

(b)

Fig. 1. a) The evolution of water depth $h(x, t)$ is shown for the dam-break problem; numerical (solid line) and exact (dotted line) solutions are nearly indistinguishable. b) The space-time plot shows where the discontinuity detector identifies a discontinuity (crosses).

\subsection{Still water test}

To test the ability of the numerical scheme to preserve the steady state of rest over varying bathymetry, the numerical solution of the SWE is computed over bathymetry given by $h_{b}(x)=a\left(b-\left(x-x_{p}\right)\right)\left(b+\left(x-x_{p}\right)\right) / b^{2}$ for $\left|x-x_{p}\right| \leq b$ and zero otherwise. The exact solution is $\mathbf{u}=0$ and $H=h+h_{b}=1 .\left(x_{p}=10\right.$, $a=0.5, b=2$, and $\mathrm{F}=1$.) The rest state is preserved up to machine precision, at least as long as $t=20$ on irregular grids. The topography and depth are approximated here with the same basis and test functions. Hence, the rest state is exactly preserved. The proof of the latter is as follows. Due to the continuous 
Table 3

\begin{tabular}{cccccc}
\hline$N \times N$ & $h$ & & & $h u$ & \\
\cline { 2 - 3 } \cline { 6 - 6 } & $L_{2}$ error & Order $p$ & & $L_{2}$ error & Order $p$ \\
\hline $20 \times 1$ & $1.059 \mathrm{e}-02$ & & & $2.545 \mathrm{e}-02$ & \\
$40 \times 1$ & $2.190 \mathrm{e}-03$ & 2.27 & & $4.546 \mathrm{e}-03$ & 2.49 \\
$80 \times 1$ & $4.188 \mathrm{e}-04$ & 2.38 & & $7.775 \mathrm{e}-04$ & 2.55 \\
$160 \times 1$ & $8.676 \mathrm{e}-05$ & 2.27 & & $1.330 \mathrm{e}-04$ & 2.54 \\
\hline
\end{tabular}

The $L_{2}$ error for $h$ and $h u$ and convergence rates are given for subcritical flow on an irregular mesh for the HLLC flux at $t=20$.

approximation of topography $h_{b}$ all variables are continuous for the rest state. Both numerical fluxes are consistent for the rest state, i.e. $\tilde{F}(U, U)=F(U)$, and therefore reduce to the real flux. Finally, the weak formulation shows that hydrostatic balance is then preserved, see also [3]. The simplicity of this result stems from our continuous approximation of topography, which approach can not be used when the bottom is discontinuous or moving discontinuously. We then refer to the more complex approaches in [9] and [11].

\subsection{Sub-and supercritical flow over an isolated ridge}

Consider the flow over an isolated parabolic ridge. By solving a cubic polynomial for given bottom topography and certain upstream Froude number, smooth sub- and supercritical solutions are found [19]. We consider the same bathymetry as in Section 4.3 with initial condition: $h+h_{b}=1, v=0$ and $u=1$ in $\Omega=20 \times 1$. The inflow is specified at the entrance of the channel at $x=0$ and extrapolated at the outflow boundary at $x=20$; it depends on the numerical flux (approximating the characteristics) to what extent this information is used. Both sub- and supercritical flows with $F=0.2$ and $F=1.9$ are considered. After reaching a steady state, accuracy errors and convergence rates are shown for the two numerical fluxes and two flow states in Tables 3 to 6 . Furthermore, the computational costs of the simulations have been calculated. Table 7 presents the absolute CPU time $T_{a}$ (in seconds) for the HLLC and kinetic numerical fluxes ( $T_{a}^{h l l c}$ and $T_{a}^{k i n}$, respectively), as well as the relative computational cost $T_{r}=T_{a}^{k i n} / T_{a}^{h l l c}$. The kinetic flux is thus more expensive for these simple test, but it turns out to be more robust in complex simulations with multiple bores. Figures $2 \mathrm{a}, \mathrm{b}$ ) show comparisons between numerical and exact solutions for the sub- and supercritical cases. 
Table 4

\begin{tabular}{cccccc}
\hline \multirow{2}{*}{$N \times N$} & $h$ & & $h u$ & \\
\cline { 2 - 3 } \cline { 6 - 6 } \cline { 5 - 6 } & $L_{2}$ error & Order $p$ & & $L_{2}$ error & Order $p$ \\
\hline $20 \times 1$ & $8.921 \mathrm{e}-03$ & & & $2.546 \mathrm{e}-02$ & \\
$40 \times 1$ & $1.836 \mathrm{e}-03$ & 2.28 & & $4.562 \mathrm{e}-03$ & 2.48 \\
$80 \times 1$ & $3.712 \mathrm{e}-04$ & 2.30 & & $7.802 \mathrm{e}-04$ & 2.54 \\
$160 \times 1$ & $6.860 \mathrm{e}-05$ & 2.43 & & $1.366 \mathrm{e}-04$ & 2.51 \\
\hline
\end{tabular}

The $L_{2}$ error for $h$ and $h u$ and convergences rate are shown for subcritical flow on an irregular mesh for the kinetic flux at $t=20$ and for the same meshes as in Table 3.

Table 5

\begin{tabular}{cccccc}
\hline$N \times N$ & $h$ & & & $h u$ & \\
\cline { 2 - 3 } \cline { 5 - 6 } \cline { 5 - 6 } & $L_{2}$ error & Order $p$ & & $L_{2}$ error & Order $p$ \\
\hline $20 \times 1$ & $4.216 \mathrm{e}-02$ & & & $1.212 \mathrm{e}-04$ & \\
$40 \times 1$ & $7.701 \mathrm{e}-03$ & 2.45 & & $4.826 \mathrm{e}-06$ & 4.65 \\
$80 \times 1$ & $1.464 \mathrm{e}-03$ & 2.39 & & $8.941 \mathrm{e}-07$ & 2.43 \\
$160 \times 1$ & $2.771 \mathrm{e}-04$ & 2.40 & & $7.983 \mathrm{e}-09$ & 6.80 \\
\hline
\end{tabular}

The $L_{2}$ error for $h$ and $h u$ and convergence rates are shown for supercritical flow on an irregular mesh for the HLLC flux at $t=60$. The superconvergence in $h u$ occurs because it is constant in steady state (after transients have disappeared).

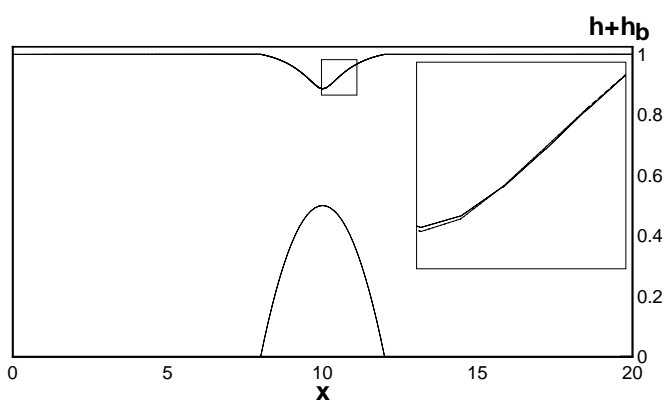

(a)

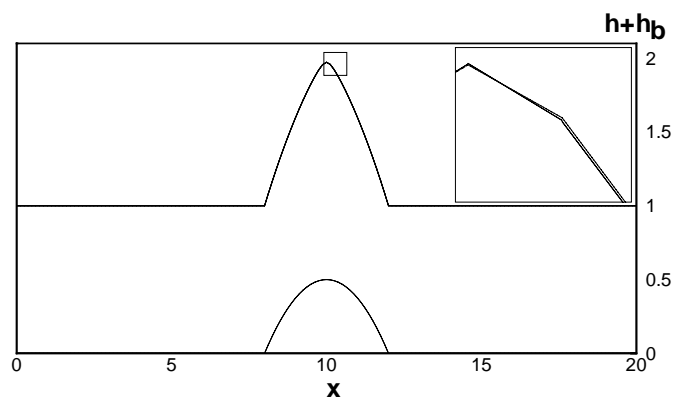

(b)

Fig. 2. Snapshots of the DG and exact solutions of the topography $h_{b}(x)$ and water surface $H(x, t)=h_{b}(x)+h(x, t)$ are shown for: a) subcritical flow (160 elements) and b) supercritical flow (160 elements) over an isolated ridge using the kinetic flux. Computations (solid lines) and exact solutions (dotted lines) are nearly indistinguishable. 
Table 6

\begin{tabular}{cccccc}
\hline \multirow{2}{*}{$N \times N$} & $h$ & & & $h u$ & \\
\cline { 2 - 3 } \cline { 6 - 6 } & $L_{2}$ error & Order $p$ & & $L_{2}$ error & Order $p$ \\
\hline $20 \times 1$ & $2.692 \mathrm{e}-02$ & & & $1.243 \mathrm{e}-03$ & \\
$40 \times 1$ & $6.026 \mathrm{e}-03$ & 2.15 & & $2.174 \mathrm{e}-04$ & 2.51 \\
$80 \times 1$ & $1.240 \mathrm{e}-03$ & 2.28 & & $4.649 \mathrm{e}-05$ & 2.22 \\
$160 \times 1$ & $2.267 \mathrm{e}-04$ & 2.45 & & $8.618 \mathrm{e}-06$ & 2.43 \\
\hline
\end{tabular}

The $L_{2}$ error for $h$ and $h u$ and convergence rates are shown for supercritical flow on an irregular mesh for the kinetic flux at $t=60$.

Table 7

\begin{tabular}{ccccccc}
\hline \multirow{2}{*}{$N \times N$} & \multicolumn{3}{l}{ Subcritical flow } & \multicolumn{3}{c}{ Supercritical flow } \\
\cline { 2 - 7 } & $T_{a}^{\text {hllc }}$ & $T_{a}^{\text {kin }}$ & $T_{r}$ & $T_{a}^{\text {hllc }}$ & $T_{a}^{\text {kin }}$ & $T_{r}$ \\
\hline $20 \times 1$ & 40 & 79 & 1.975 & 146 & 217 & 1.486 \\
$40 \times 1$ & 93 & 134 & 1.440 & 264 & 448 & 1.696 \\
$80 \times 1$ & 160 & 287 & 1.790 & 542 & 818 & 1.501 \\
$160 \times 1$ & 692 & 1142 & 1.650 & 1097 & 1709 & 1.550 \\
\hline
\end{tabular}

Computational performance of the subcritical and supercritical flow tests.

\section{$5 \quad$ Validation of the model}

From (2.8), we see that PV is generated when the dissipation $E_{D}$ varies along the bore. In the absence of bores, PV is materially conserved. Furthermore, since $h \Pi=\partial_{x} v-\partial_{y} u$ is the vorticity, sheared flows are vortical flows. The generation of PV is most clearly illustrated when the initial flow has zero vorticity. In the absence of another source of dissipation than the one in bores, PV can only be generated through non-uniform shallow-water wave breaking represented by these bores. The following three simulations aim to illustrate the PV generation mechanism. In all these examples, the expression (2.8) allows us to qualitatively predict the ensuing vortical flows as the associated shear profile or signature of the eddies generated. Once the PV is generated it is actively advected by the flow.

The following three cases are considered. (i) A linear normal mode solution of a gravity wave is used as initial condition in simulations of the nonlinear flow. Given these linear solutions at time zero, $u_{0}(\mathbf{x}), v_{0}(\mathbf{x})$ and $\eta_{0}(\mathbf{x})$, the initial condition of the nonlinear flow is $u_{0}(\mathbf{x}), v_{0}(\mathbf{x})$ and $h_{0}(\mathbf{x})=H+\eta_{0}(\mathbf{x})$ with $H$ the constant still water depth and $\eta_{0}(\mathbf{x})$ the initial departure of the free surface from it. In addition, both for the linear and nonlinear flow, the PV is zero initially and remains zero unless $\mathrm{PV}$ is generated through non-uniform bore 


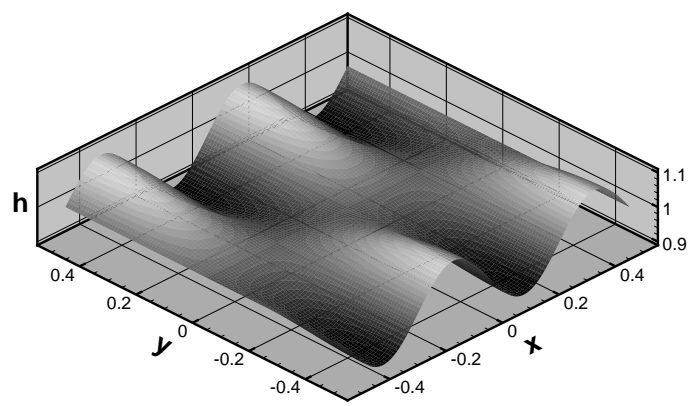

(a)

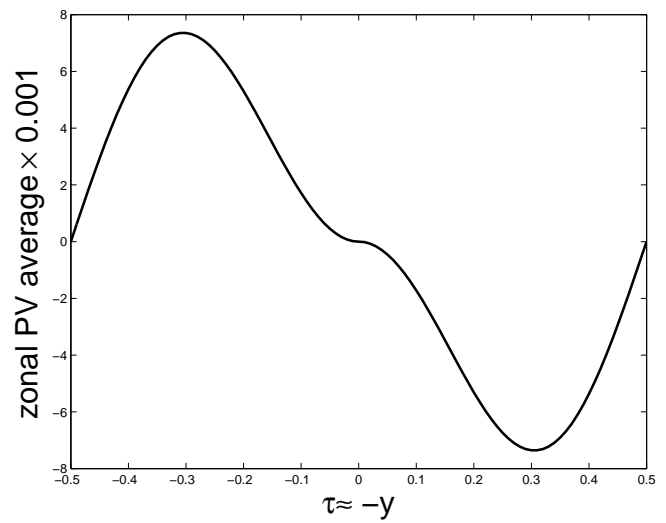

(b)

Fig. 3. (a) Initial condition for the test with steepening waves and bores in a channel with a resolution of $100 \times 100$ elements. (b) We show an estimate of the PV generated by a non-uniform bore based on formulas (2.8) and (5.1). The along-bore coordinate $\tau$ is roughly opposite to $y$.

formation. Given a periodic channel in the $x$ direction, the average potential vorticity $q=\bar{\Pi}(y, t)$ and shear $\bar{u}(y, t)$, both averaged in the $x$ direction, signal a transfer from the zero PV gravity waves to the vortical flow. (ii,iii) Uniform bores are simulated to encounter non-uniform topographical features, such that the non-uniform bores emerge and generate PV in the form of eddies whose signs we predict. Peregrine [31] sketches qualitatively how bores of finite length or pierced in the middle generate eddies. In particular, we simulate flow of an initially uniform bore over (ii) conical and (iii) Gaussian topography to compare with Hu's results [20].

\subsection{Shallow water waves and bores in a channel}

Nonlinear wave breaking is investigated by initializing the flow with a gravity wave solution of the linearized shallow water equations, in which $h(\mathbf{x}, 0)=$ $H+A \sin (l y) \sin k x$ with $l=(2 n+1) \pi / L_{y}, k=2 \pi m / L_{x} ;$ and, $\mathrm{F}=H=$ $1, A=0.1$ and $n=0, m=2$. The domain is a channel of size $L_{x} \times L_{y}=1 \times 1$ with a flat bottom, solid walls at $y= \pm 0.5$, and periodic boundary conditions in the zonal direction $x$. The $\mathrm{PV}$ is initially zero. We expect the initially harmonic waves to steepen, break into bores, and subsequently dampen due to the localized energy dissipation (2.7) at the bores. Free surface profiles and PV contours are shown at times $t=0.2,0.5, \ldots, 15$ in Figures 4 to 5 . The wave peaks depicted in Figure 3a) appear to be preserved in the depth profiles, shown at subsequent times $t=0.2$ and $t=0.5$ in Figures $4 \mathrm{a}$ ) and $4 c)$, respectively. PV should be zero before wave breaking starts; what is seen at $t=0.0$ and $t=0.2$ are second-order truncation errors. This suggest the 
following estimate of $h_{1}$ and $h_{2}$ from the initial condition given above

$$
h_{2}=H+A \sin (l|\tau|) \quad \text { and } \quad h_{1}=H-A \sin (l|\tau|)
$$

with $-0.5<\tau<0.5$ and $\tau$ aligned roughly opposite to $y$ as the bore is traveling in the negative $x$-direction. Although the bores will commence near the walls, they are seen to grow into the center of the channel. Based on our rough estimates (5.1) and (2.8), we calculate the approximate PV profile given in Figure $3 \mathrm{~b})$. We expect the formation of two jets after many wave periods with a negative zonal average $\bar{u}(y, t)<0$ for $y>0(\tau<0)$, since PV is positive, and negative for $y<0(\tau>0)$. Note that the harmonic waves specified as initial condition have zero zonal average. The estimated profile of PV in Figure 3 is recognized in the early calculated profiles of zonally averaged PV in Figure 6. A rough estimate of the time $t_{b}$ of wave breaking is given by using the initial profile for one characteristic, which yields the Burgers' equation estimate $t_{b} \approx 0.53$. For smooth flows in the zonally periodic channel the energy $\mathrm{E}(t)=\frac{1}{2} \int_{\Omega_{\mathrm{h}}} h|\mathbf{u}|^{2}+\mathrm{F}^{-2} h^{2} d \Omega=\sum_{K} \frac{1}{2} \int_{K_{\mathrm{h}}} h|\mathbf{u}|^{2}+\mathrm{F}^{-2} h^{2} d \Omega$ is conserved. Energy is plotted from time $t=0$ to $t=25$ in Figure 7a). It is seen to be approximately conserved before the onset of breaking around $t=0.5$ : thereafter energy is dissipated in the non-uniform bores. Once the bores have disappeared, the energy should be conserved exactly again, which is approximately the case in the numerics for large times $(t \gtrsim 15)$. In Figure $7 \mathrm{~b})$ zonally averaged profiles $\bar{u}(y, t)$ of the velocity are shown as function of $y$ and $t$. Two jets eventually form near the walls.

\subsection{Flow over a conical hump}

The passage of a bore over a conical hump is considered, cf. Matsutomi and Mochizula [27] and $\mathrm{Hu}$ [20]. The bore is generated via a dam break and the geometry is given in Figure 8. The isolated conical shaped hump of height $h_{b v}$ and radius $r_{v}$,

$$
h_{b}(\mathbf{x})= \begin{cases}h_{b v}-\frac{h_{b v}}{r_{v}} \sqrt{\left(x-x_{v}\right)^{2}+\left(y-y_{v}\right)^{2}} & \text { if }\left(x-x_{v}\right)^{2}+\left(y-y_{v}\right)^{2} \leq r_{v}^{2} \\ 0 & \text { otherwise }\end{cases}
$$

is located in a rectangular domain $\Omega=\left[L_{x} \times L_{y}\right]$ and centered at $\left(x_{v}, y_{v}\right)$. The initial condition is given by a discontinuity in the water surface $h(\mathbf{x}, 0)=h_{l}$ for $x<x_{0}$ and $h(\mathbf{x}, 0)=h_{r}$ for $x \geq x_{0}$ and fluid initially at rest, $\mathbf{u}(\mathbf{x}, 0)=0$. Parameter values are $h_{b v}=0.012, r_{v}=1.2,\left(x_{v}, y_{v}\right)=(2.5,1.3), L_{x}=4, L_{y}=$ 2.6, $x_{0}=1, h_{l}=0.09, h_{r}=0.02$, and $\mathrm{F}=1$. All boundaries are considered to be solid walls except an open boundary at $x=4$. After the collapse of the dam, a bore is formed and propagates downstream over the conical hump. A qualitative prediction of the PV generation follows from (2.8). Bore dissipation 


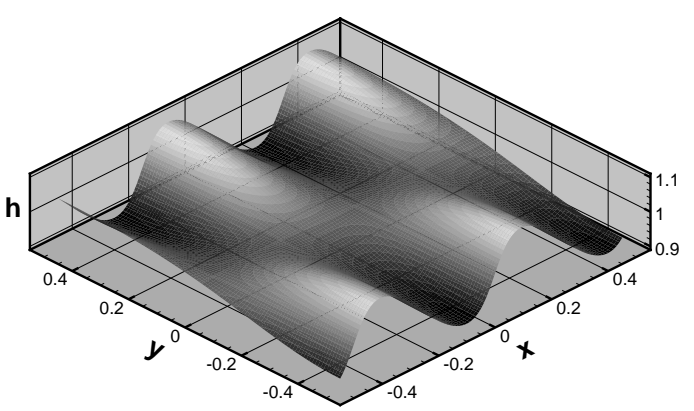

(a)

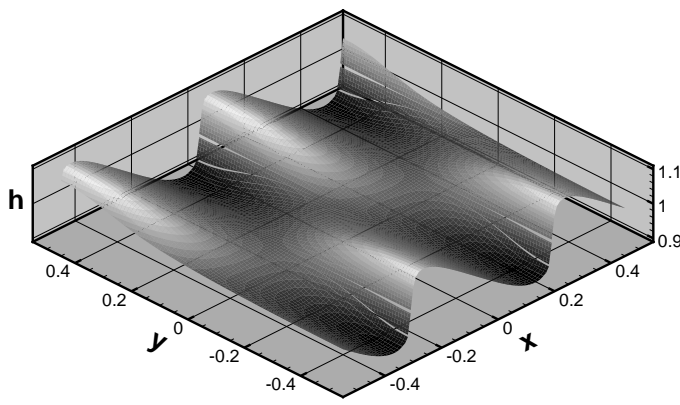

(c)

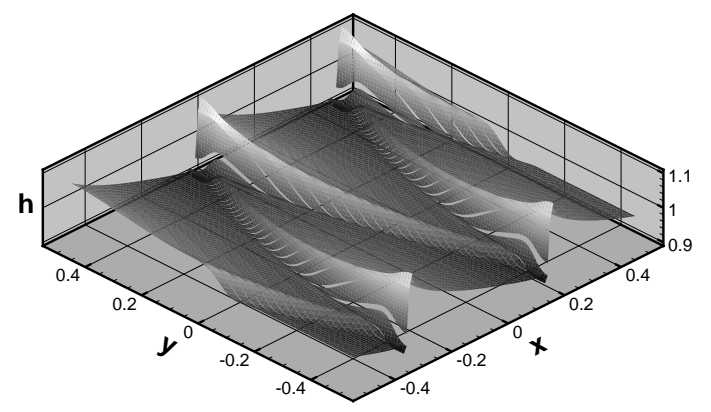

(e)

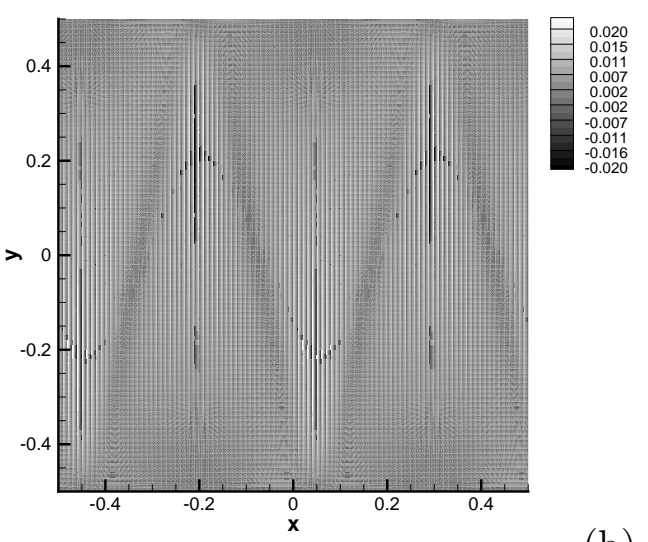

(b)

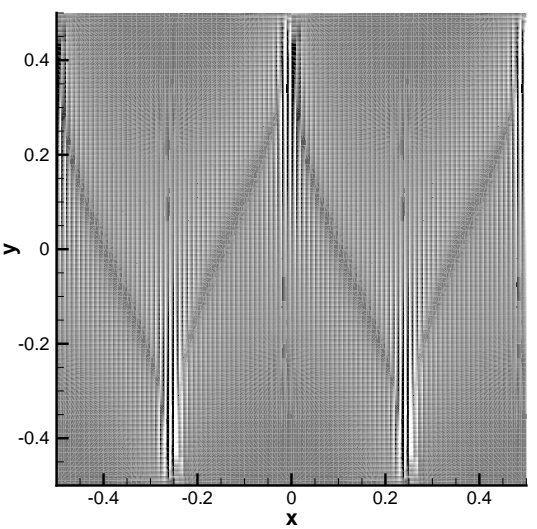

(d)

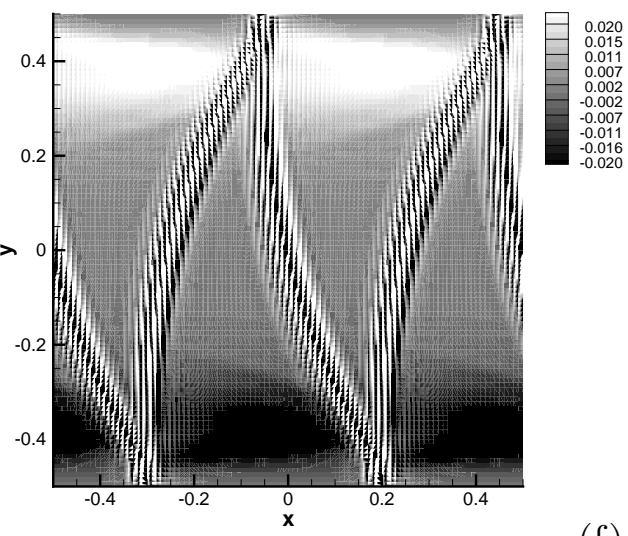

(f)

Fig. 4. a) Numerical solution of $h(\mathbf{x}, t)$ and b) PV for steepening waves in a channel at time $t=0.20$. Waves travel in the minus $x$-direction. The period of corresponding harmonic waves is 0.49. c) Numerical solution of $h(\mathbf{x}, t)$ and d) PV for breaking waves in a channel at time $t=0.50$. Bore formation has begun. (e,f) Same at time $t=2$. Before breaking, just begun near the walls at $t=0.5, \mathrm{PV}$ should be zero, but as it is not preserved numerically some PV noise is visible in (b) and (d). This noise will stay visible around the bores in (f), where the numerically solution limits numerical oscillations, which nevertheless locally hamper the calculation of PV as it is a derived quantity from our prime flow variables. 


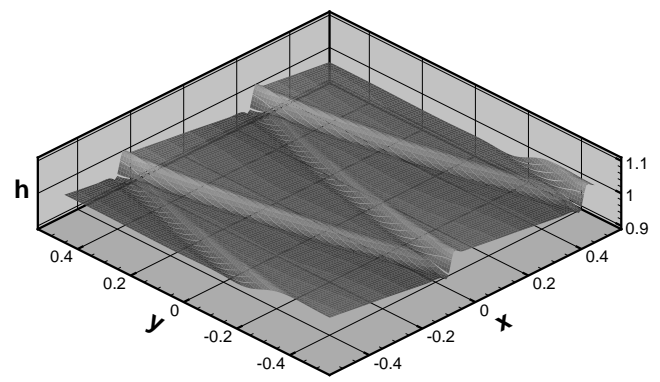

(a)

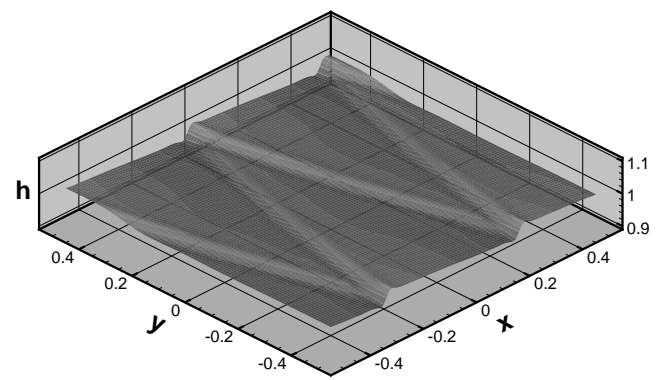

(c)
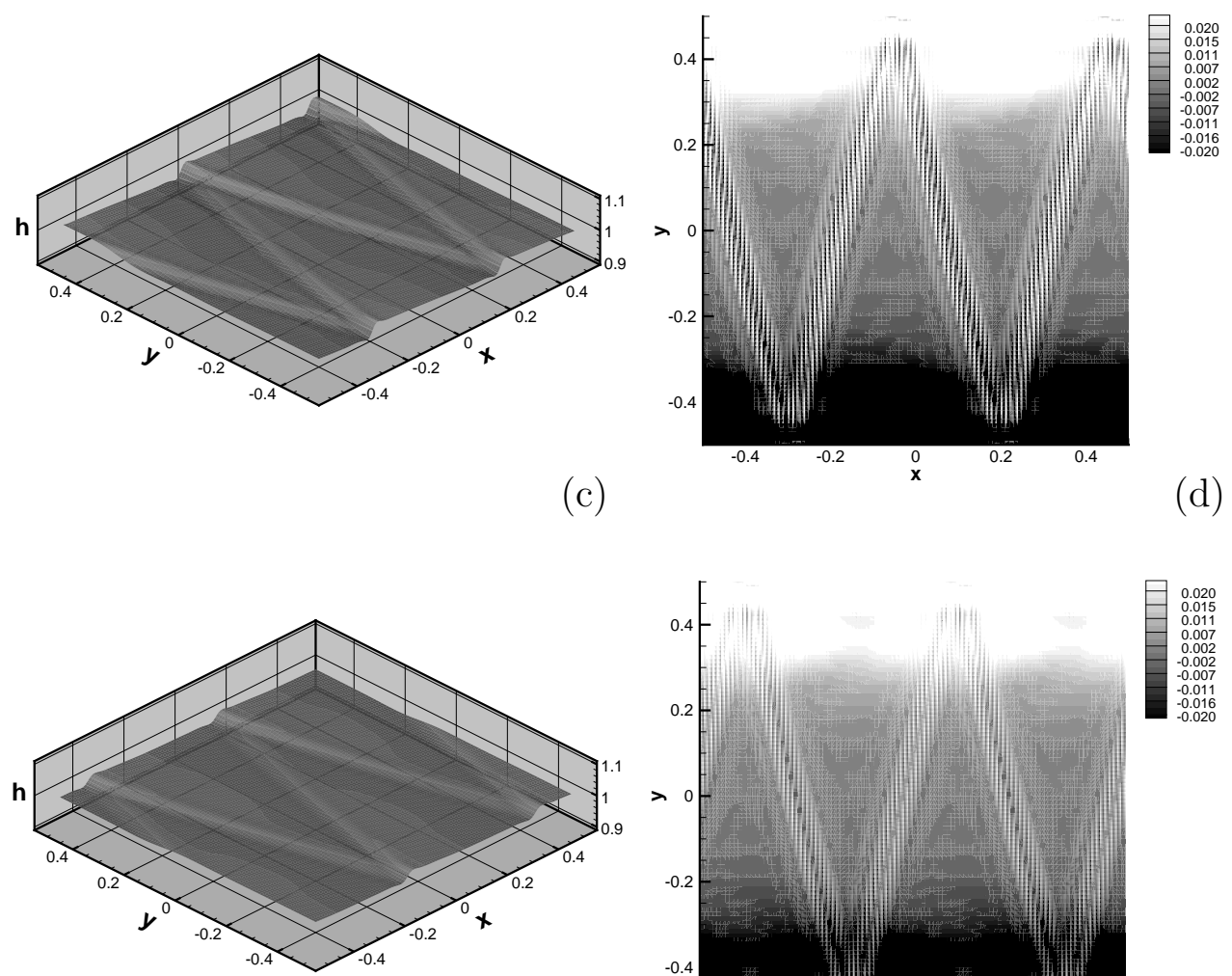

(e)

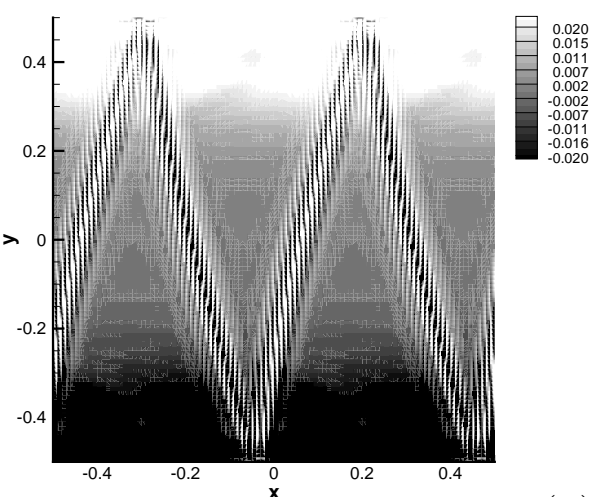

(b)

(d)
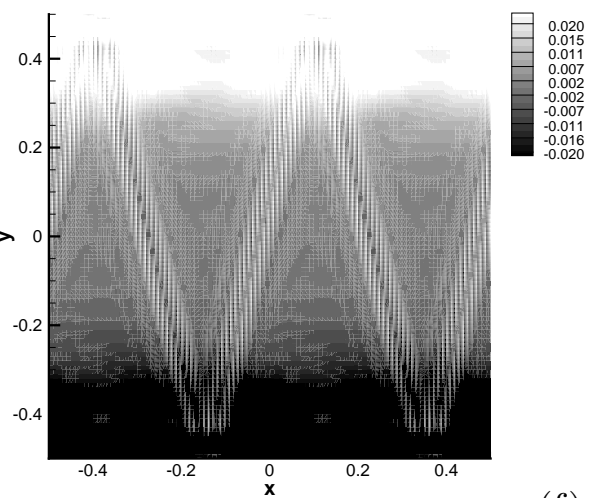

(f)

Fig. 5. a) Numerical solution of $h(\mathbf{x}, t)$ and b) PV in a channel at time $t=8$. (c,d) Same at time $t=15$. (e,f) Same at $t=25$. PV noise stays visible around the bores in $(b, d, f)$.

is higher in the shallower waters over the conical hump. Consider a bore aligned approximately in the $y$-direction. We and also Ambati and Bokhove [3] observe from their simulations that the free surface remains rather flat, which allows a more quantitative analysis of (2.8). For $y<0$ and $\tau<0, \partial E_{D} / \partial y>0$ and vice versa for $y>0$ and $\tau>0$. Hence, a positive PV anomaly is expected for $y>0$ and a negative one for $y<0$, which are symmetric in shape due to the 


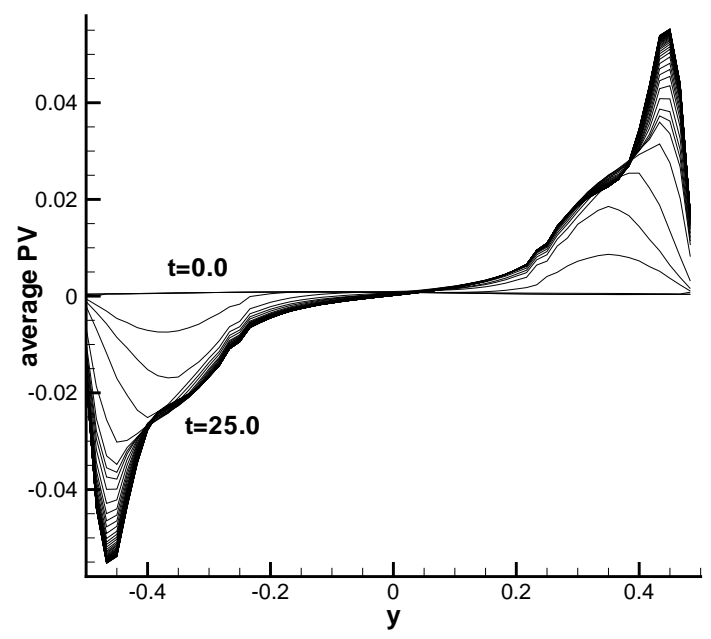

Fig. 6. The zonal average of the PV, defined by $q(y, t)=\bar{\Pi}(\mathbf{x}, t)$, is shown as function of $y$ and $t$.

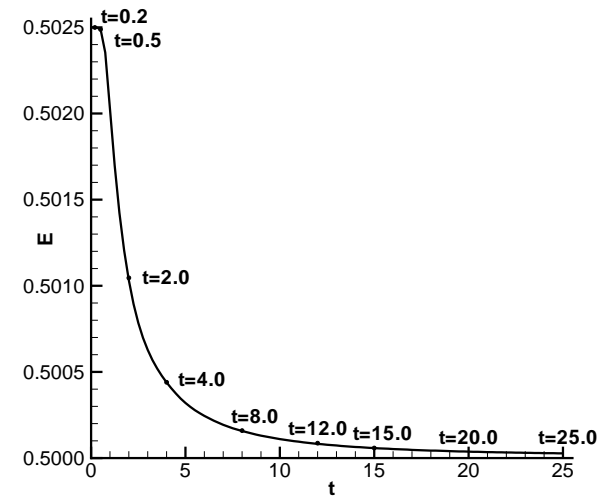

(a)

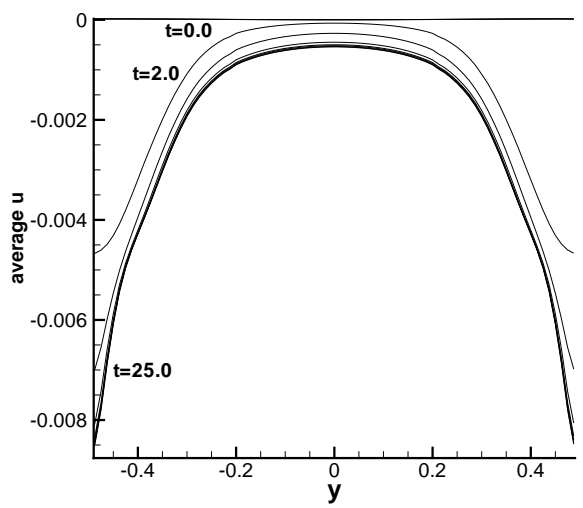

(b)

Fig. 7. (a) The global energy, the sum of kinetic and potential energy, is shown as function of time. Before bores emerge, the energy should be conserved exactly, which is approximately the case in the numerics. After bore formation, energy is dissipated in the bores, which is clearly visible after $t \approx 0.5$, till the bores have dissipated at large times. (b) The zonal average of the velocity $u$, defined by $\bar{u}(y, t)$, is shown as function of $y$ and $t$ at times $0,0.2,0.5,2,4,8,12,15,20,25$.

symmetry of the conical hump. After the PV anomaly has been generated, it is advected along. Figure 9 shows that the evolution of the PV - at times $t=$ $0,2,4,6,8$ and $t=10$ - confirms our qualitative prediction, and is similar to the simulations of $\mathrm{Hu}$ [20], who used LeVeque's finite volume scheme [26]. The numerical noise in our simulation, caused by the discontinuous first derivative of the topography, is less than in [20]. The global vorticity or weighted PV should remain zero, which is approximately true in the simulations. Finally, 


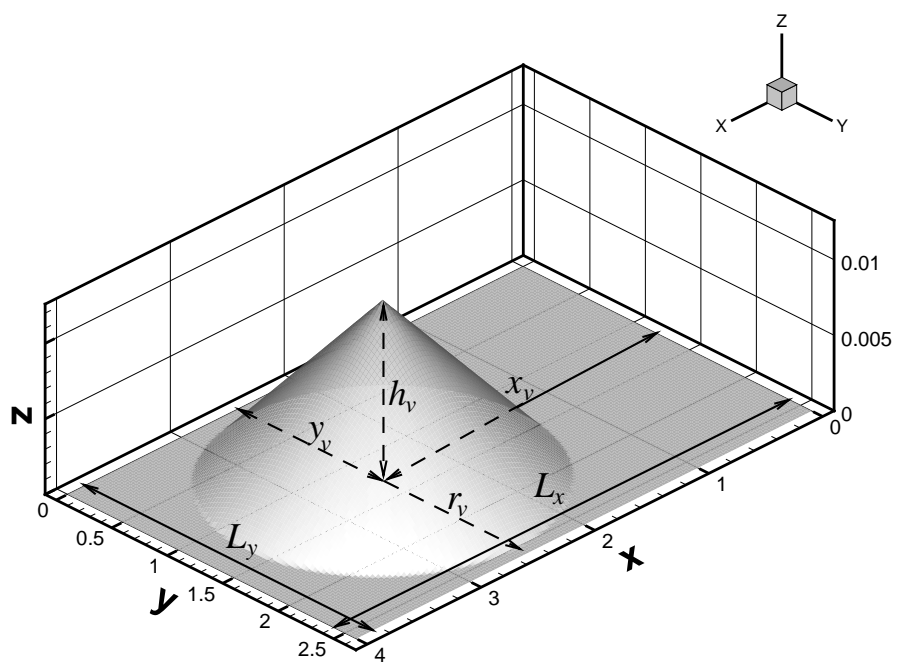

Fig. 8. The geometry is shown for the conical hump experiment.

we display the surface profiles at various times in Fig. 10; these compare well with the experiments of Matsutomi and Mochizula [27] and $\mathrm{Hu}$ [20].

\subsection{Flow over a Gaussian hump}

In this test, a bore forms after a dam breaks in a rectangular domain $\Omega=$ $\left[L_{x} \times L_{y}\right]$ with bottom topography consisting of a isolated Gaussian hump defined by

$$
h_{b}(\mathbf{x})=h_{b v} e^{\left(-12.5\left(x-x_{v}\right)^{2}-12.5\left(y-y_{v}\right)^{2}\right)},
$$

centered at $\left(x_{v}, y_{v}\right)$ and with maximum height $h_{b v}$. The initial condition is given by $h(\mathbf{x}, 0)=h_{l}$ for $x<x_{0}$ and $h(\mathbf{x}, 0)+h_{b}(\mathbf{x})=h_{r}$ for $x \geq x_{0}$ and fluid initially at rest, $\mathbf{u}(\mathbf{x}, 0)=0$. Parameter values are $h_{b v}=0.015,\left(x_{v}, y_{v}\right)=$ $(1.75,1.75), L_{x}=L_{y}=3.5, x_{0}=0.5, h_{l}=0.11, h_{r}=0.02$, and $\mathrm{F}=1$. Boundary conditions are the same as in the conical hump test, but the exit of the channel is located at $x=3.5$. After the collapse of the dam, a bore is formed and propagates downstream over the Gaussian hump. The generation of two initial eddies and their signs, in Figure 11 at times $t=4$ and 6 , has the same explanation as in the previous test of flow over a conical hump. Figure 11 at times $t=8$ and $t=10$ shows the formation of two new eddies arising from a reflected bore. In Figure 12, a second hydraulic jump is seen to develop at time $t=8$ as the bore propagates downstream. The bore propagates against the current with maximum dissipation along the centerline $y \approx 0$ where waters are most shallow, so $y<0$ corresponds to $\tau>0$ and $\partial E_{D} / \partial \tau<0$ and vice versa for $y>0$ and $\tau<0$. Hence, for the second group of eddies, formation of a negative PV anomaly is expected for $y<0(\tau>0)$ and a positive one for $y>0(\tau<0)$. Our simulations confirm these and Hu's results [20]. 

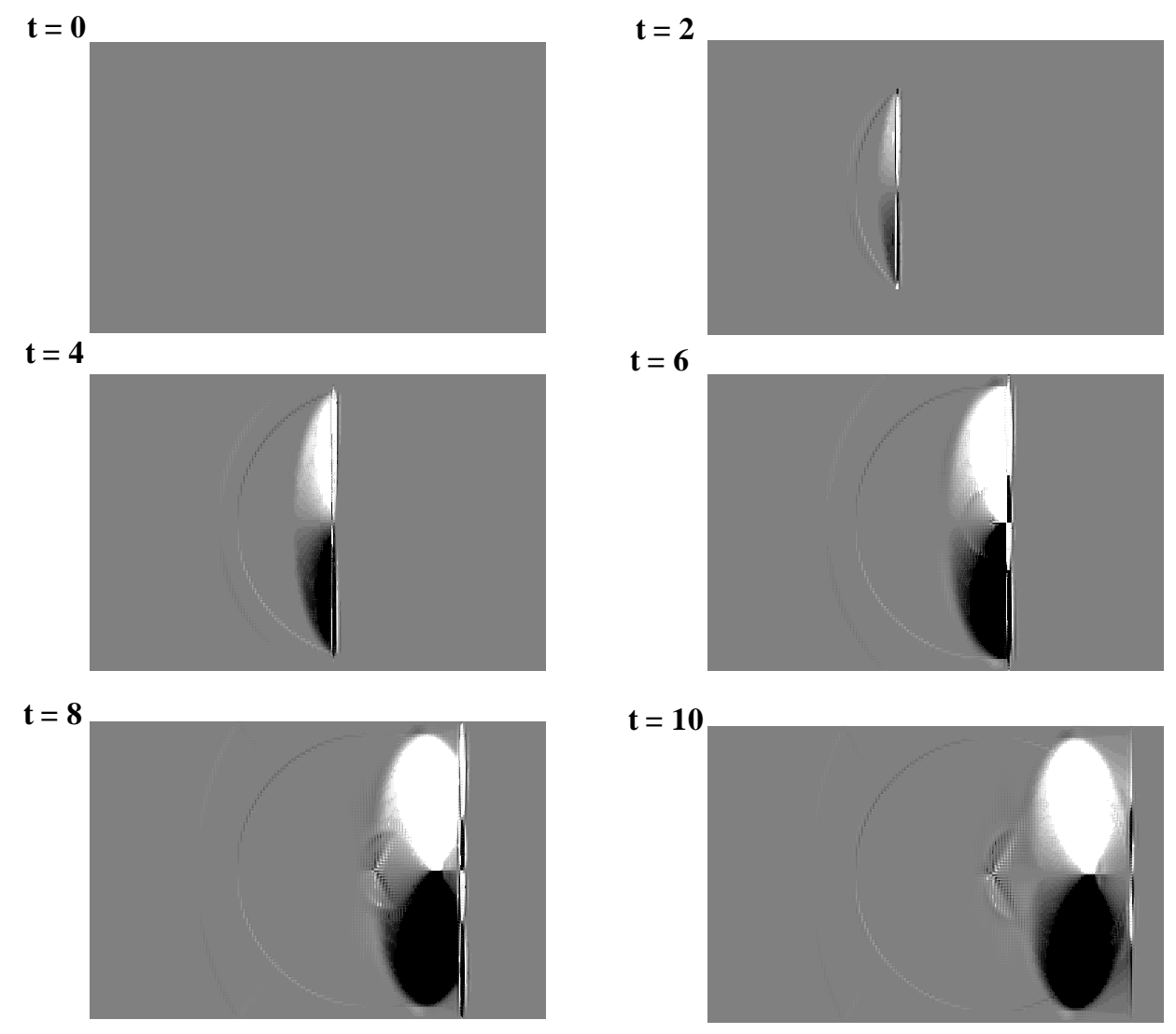

$\begin{array}{lllllllllll}-0.50 & -0.39 & -0.29 & -0.18 & -0.08 & 0.03 & 0.13 & 0.24 & 0.34 & 0.45 & 0.50\end{array}$

Fig. 9. Contours of PV generated by a dam break flow over a conical hump. We used $160 \times 100$ elements.

\subsection{Oblique hydraulic jumps in flow through a contraction}

Theoretical [2] and experimental results [1] reveal the development of steady oblique hydraulic jumps in narrow flumes with a contraction. From the shock relations of the shallow water system (2.3) and (2.4), the jump ratio $h_{1} / h_{0}$ across the shock and the angle $\theta_{s}$ of the shock can be determined by the following equations [2]:

$$
\frac{h_{1}}{h_{0}}=\frac{\tan \theta_{s}}{\tan \left(\theta_{s}-\theta_{c}\right)} \quad \text { and } \quad \sin \theta_{s}=\sqrt{\frac{1}{2 \mathrm{~F}^{2}} \frac{h_{1}}{h_{0}}\left(1+\frac{h_{1}}{h_{0}}\right)},
$$

where $h_{0}$ and $h_{1}$ are the upstream and downstream depths across the oblique hydraulic jump, and $\theta_{c}$ and $\theta_{s}$ are the angles of the contraction and the shock measured relative to the horizontal wall of the flume, respectively. To assess the convergence to steady state and to compare the HLLC and kinetic flux, we perform simulations of two-dimensional flow through a flume with a contraction with a scaled minimum width $b_{c}$ and a constant upstream 


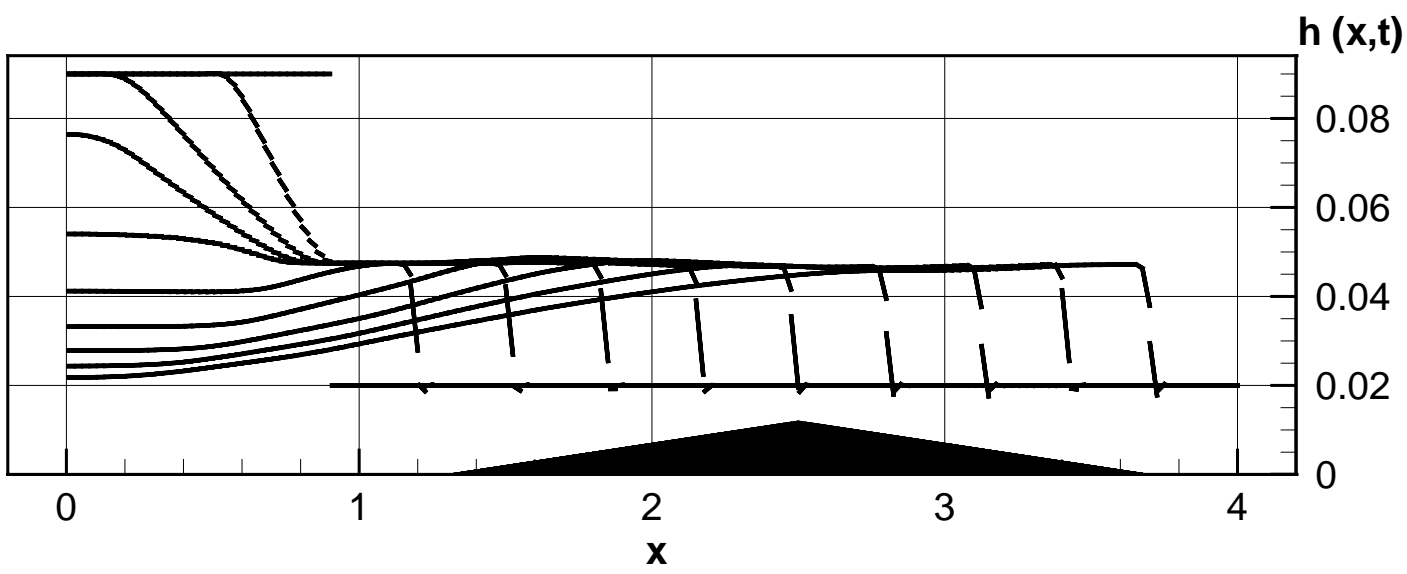

Fig. 10. Surface profiles of $h(\mathbf{x}, t)$ are shown in the middle of the channel at times 0.0 to 10.0 with an increment of 1.0. These profiles compare favorably with simulations of $\mathrm{Hu}$ [20] and laboratory experiments of Matsutomi and Mochizula [27].

width $b_{0}=1$. The initial condition was a flow at rest with a small constant depth $h(x, 0)=0.001$. Simulations were run to steady state for a Froude number $\mathrm{F}=3.65$, outflow and solid wall boundary conditions, and inflow with $h(0, t)=1, u(0, t)=1, v(0, t)=0$. To avoid negative water depths, the slopes in the numerical approximation of water depth were set to zero when the depth becomes smaller than $h(x, 0)$. The computational domain, nondimensionalized with the flume width $l_{0}^{*}=20 \mathrm{~cm}$, consists of an inlet section, a contraction section, and an outlet section, see Fig. 13a). This configuration naturally enforces a critical condition at the point of minimum width, at which point in the experiment the water falls into a bucket. The length of the contraction along the wall is 1.5. For $b_{c}=0.6$, theoretical calculations yield a jump ratio $h_{1} / h_{0}=1.5463$ and a jump angle $\theta_{s}=22.608^{\circ}$, and for $b_{c}=0.7$, $h_{1} / h_{0}=1.4019$ and $\theta_{s}=20.824^{\circ}$. The convergence to steady state has been considered for simulations on four different meshes. Details of each mesh are given in Table 8 as well as the convergence rates to the exact shock angle and jump ratio.

Figure 13a) shows the steady oblique jumps at time $t=7.0$. A similar result was obtained experimentally by Akers [1] as shown in Figure 13b). The computational cost of simulations is presented in Table 9 and shows ultimately that runs with the kinetic flux are faster for both second and third order Runge-Kutta methods. While the kinetic flux is slower per time step, it allows the use of larger time steps. The same steady state results were reached in all these simulations. 

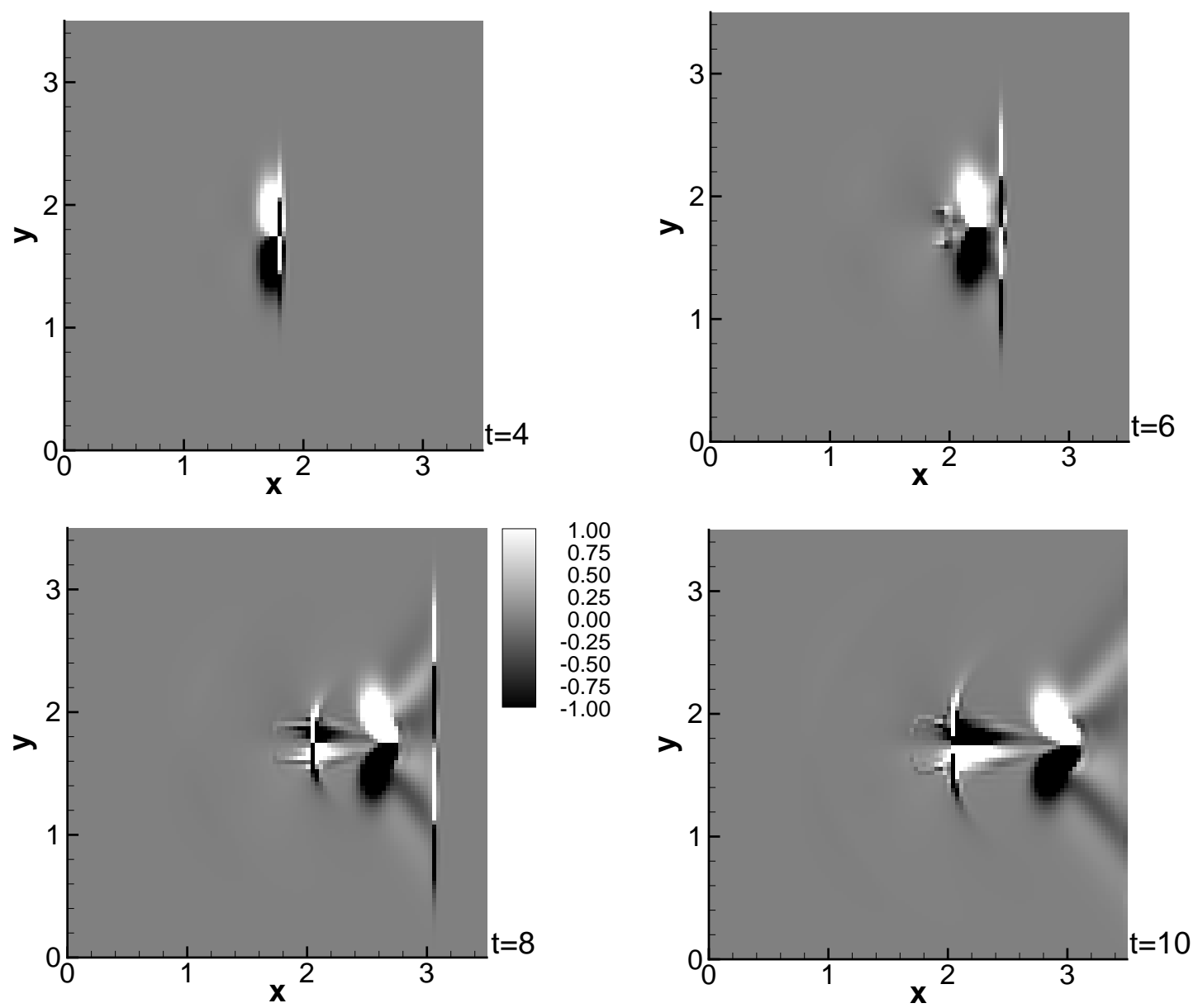

Fig. 11. Contours of PV generated by a dam break flow over a Gaussian hump. We used $100 \times 100$ elements.

\begin{tabular}{lccccccc}
\hline \multirow{2}{*}{ Mesh } & \multicolumn{6}{l}{ Flow through a contraction } \\
\cline { 2 - 7 } & Inlet & Contraction & Outlet & $b_{c}$ & $\theta_{c}$ & $\theta_{s}$ & $h_{1} / h_{0}$ \\
\hline$I$ & $10 \times 10$ & $10 \times 10$ & $10 \times 5$ & 0.6 & $7.54^{\circ}$ & $22.96^{\circ} \pm 0.72^{\circ}$ & $1.551 \pm 0.007$ \\
$I I$ & $20 \times 20$ & $20 \times 20$ & $20 \times 10$ & 0.6 & $7.54^{\circ}$ & $22.76^{\circ} \pm 0.20^{\circ}$ & $1.551 \pm 0.005$ \\
$I I I$ & $40 \times 40$ & $40 \times 40$ & $40 \times 20$ & 0.6 & $7.54^{\circ}$ & $22.70^{\circ} \pm 0.08^{\circ}$ & $1.551 \pm 0.001$ \\
$I V$ & $40 \times 40$ & $40 \times 40$ & $40 \times 20$ & 0.7 & $5.65^{\circ}$ & $20.85^{\circ} \pm 0.08^{\circ}$ & $1.400 \pm 0.001$ \\
\hline
\end{tabular}

Table 8

Computational meshes used for flow through a contraction and numerical values of $\theta_{s}$ and $h_{1} / h_{0}$. 

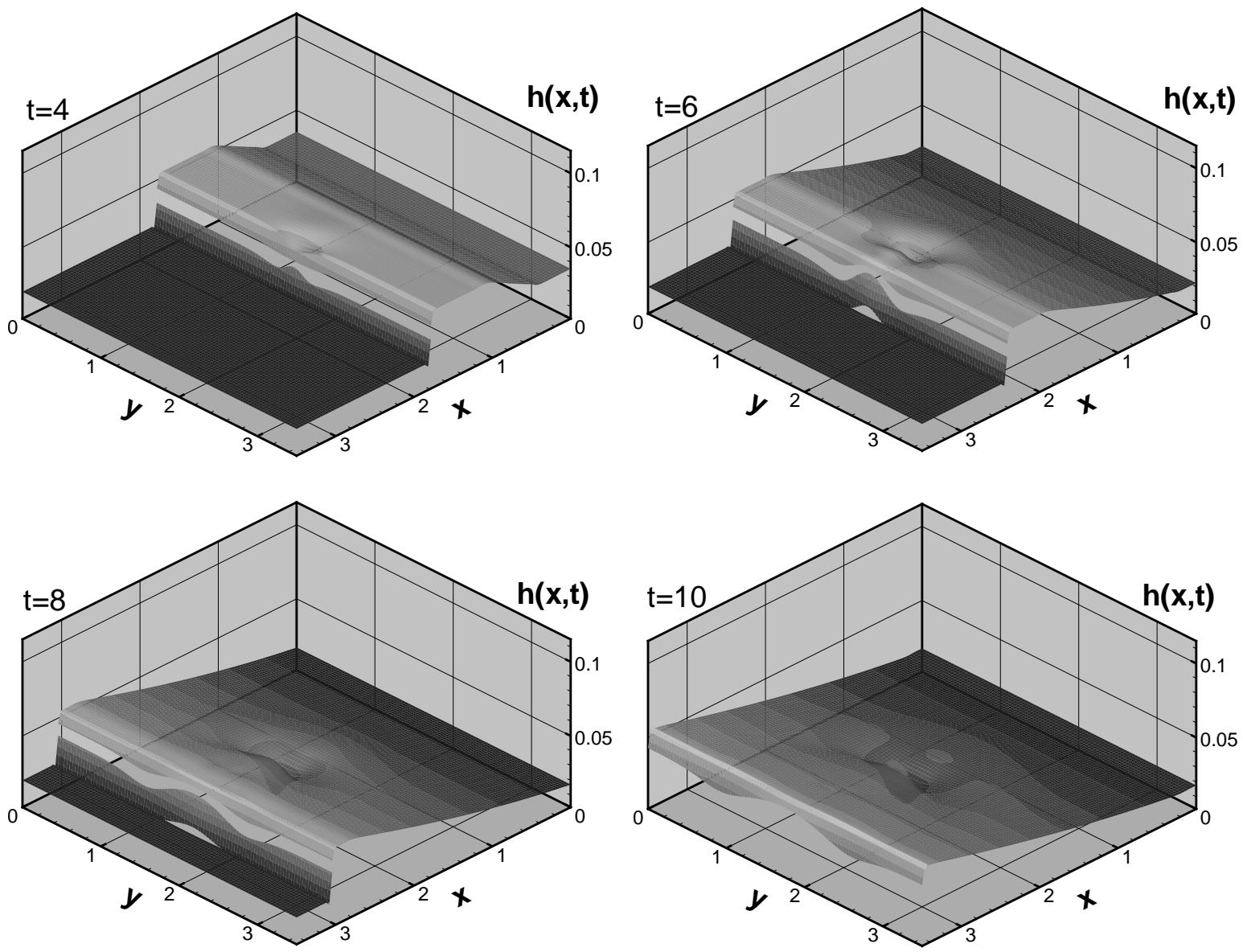

Fig. 12. Free surface profiles at times 4.0,6.0, 8.0 and 10.0.

Table 9

\begin{tabular}{lccccccc}
\hline \multirow{2}{*}{ Mesh } & \multicolumn{3}{c}{ Runge-Kutta 2 } & \multicolumn{4}{c}{ Runge-Kutta 3 } \\
\cline { 2 - 7 } & $T_{a}^{\text {hllc }}$ & $T_{a}^{\text {kin }}$ & $T_{r}$ & $T_{a}^{\text {hllc }}$ & $T_{a}^{\text {kin }}$ & $T_{r}$ \\
\hline I & 89 & 124 & 1.393 & 134 & 291 & 2.171 \\
II & 687 & 531 & 0.772 & 1540 & 938 & 0.609 \\
III & 6460 & 2195 & 0.339 & 13287 & 3742 & 0.281 \\
\hline
\end{tabular}

Computational performance for the simulated flow through a contraction using the second and third order Runge-Kutta time integration methods.

\section{Conclusions}

A discontinuous Galerkin finite element discretization has been presented for shallow water flows over varying bathymetry. This discretization had the fol- 


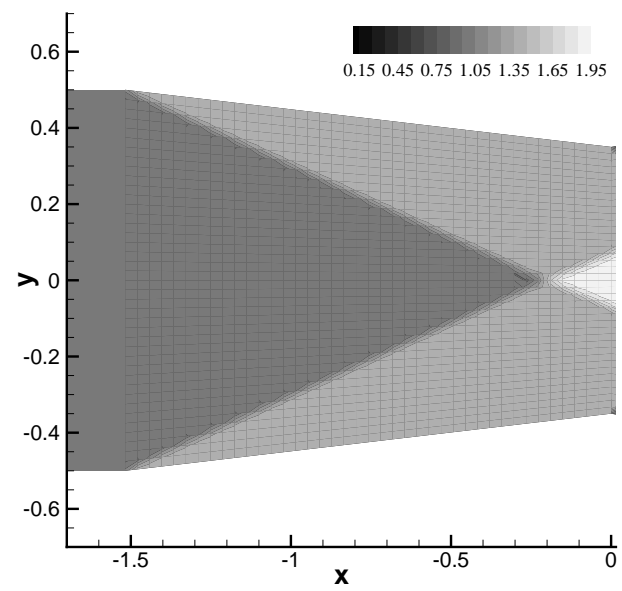

(a)

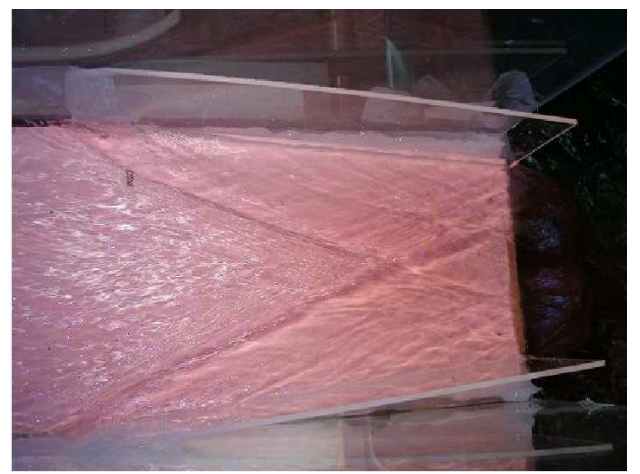

(b)

Fig. 13. a) Numerical results of steady oblique hydraulic jumps in a channel with a contraction for mesh $I V$. b) Experimental results of steady oblique hydraulic jumps in a channel with a contraction, with Froude number $F=3.65$ at the inlet and minimum contraction width $b_{c}=0.7$ at the critical point and outlet in the experiment. Measured jump ratio is unknown and angle $\theta_{s}=22.0^{\circ} \pm 1^{\circ}$; courtesy Ben Akers (Akers, 2005).

lowing novel aspects. First, two accurate numerical fluxes were presented and compared, the HLLC numerical flux [5] and a kinetic flux [33]. While the HLLC flux is a direct approximation to the Riemann problem in a Godunov-type approach at second order in the absence of bathymetry, the kinetic approach directly and more consistently includes the bathymetric terms. The kinetic approach yields a spatial discretization of the relevant Vlasov equation, which is subsequently transformed to the discretized hydrodynamic equations, using the transformation between the partial differential equations of the kinetic and SWE. The resulting weak formulations differ only with respect to the numerical flux, and both approaches can be extended to higher order. Second, the bathymetry was projected on the quadrilateral elements with the usual second-order finite element basis functions such that the resulting discretized bathymetry was continuous. In addition, the flow variables were also projected using these four basis functions. Consequently, the rest flow state can be preserved exactly in the discretization and to machine precision in the implementation. On triangles, this preservation can be achieved directly with the usual linear basis functions. The verification of numerical and exact solutions showed the methods to be second order in spatial accuracy using equal-order interpolation for the approximation of the flow variables.

The shallow water equations globally and locally preserve mass, energy, and mass weighted functions of the potential vorticity (PV) for smooth flows and appropriate boundary conditions. The PV is also a materially conserved quantity consisting of the total vertical vorticity, $\partial_{x} v-\partial_{y} u$, divided by the depth. In 
the presence of non-uniform bores, PV anomaly can be generated (cf. section 2.1 and [30]). By construction, our numerical method only conserves momentum (for $h_{b}$ constant) and mass exactly. We therefore validated simulations of bore-vortex interactions against a qualitative and asymptotic analysis of the generation of potential vorticity (PV) anomalies. Consequently, we could predict the signs of the emerging vortices and shear flows. The latter validation is important because numerical schemes may generate vortices of incorrect shape and sign, as was shown by $\mathrm{Hu}[20]$. The counterpart in compressible flows would be the (in)correct generation of entropy by non-uniform shocks. We considered, in particular, bore-vortex interactions of breaking waves in a channel and the flow of a bore over conical and Gaussian topography. The emerging vortices simulated in the flow over the conical and Gaussian hump compared favorably with simulations of $\mathrm{Hu}$ [20], performed with LeVeque's finite volume scheme [26], and laboratory experiments of Matsutomi and Mochizula [27].

Final simulations compared the numerical fluxes for oblique hydraulic jumps in a contraction, also with a laboratory experiment [1]. The kinetic flux turned out to be more robust when shocks are involved. Our next step in DG shallow water modeling for river hydraulics is the implementation of an efficient and accurate flooding and drying scheme, and the coupling of the hydrodynamics to the sediment transport.

Acknowledgments: P.T. and O.B. gratefully acknowledge a two-year scholarship of the $\mathrm{EU} \mathrm{Al} \beta$ an program and a five-year fellowship of The Royal Netherlands Academy of Arts and Sciences (KNAW), respectively. Financial assistance from Conicet (Argentina) is also gratefully acknowledged by P.T. and C.V. We kindly thank Professor Howell Peregrine for his insightful comments on PV generation by bores and for pointing out an error. We thank the anonymous reviewers for their valuable remarks.

\section{Appendices}

\section{A Proof of proposition 3.1}

From (3.18) and (3.19) we find that

$$
h=K G^{2}=\int \mathrm{Dd} \bar{\zeta} \quad \text { and } \quad\left(\sigma u_{i}\right)=\mu_{i}=K G^{2} u_{i}=\int \zeta_{i} \mathrm{Dd} \bar{\zeta} .
$$


Multiply (3.17) by the vector $(1, \bar{\zeta})^{T}$ and integrate over $\bar{\zeta}$ to obtain

$$
\int\left[\partial_{t} \mathrm{D}\left(\begin{array}{l}
1 \\
\bar{\zeta}
\end{array}\right)+\nabla \cdot(\bar{\zeta} \mathrm{D})\left(\begin{array}{l}
1 \\
\bar{\zeta}
\end{array}\right)-\nabla_{\zeta} \cdot\left[\left(\mathrm{F}^{-2} \nabla h_{b}\right) \mathrm{D}\right]\left(\begin{array}{l}
1 \\
\bar{\zeta}
\end{array}\right)\right] \mathrm{d} \bar{\zeta}=0 .
$$

Evaluating the integrals in (A.2) while using definition (3.19), (A.1), the symmetry properties (3.18) of $\chi$, integration by parts, and (3.20), we find

$$
\begin{gathered}
\int \mathrm{D}(1, \bar{\zeta})^{T} \mathrm{~d} \bar{\zeta}=(h, h \mathbf{u})^{T} \\
\int \boldsymbol{\nabla} \cdot(\bar{\zeta} \mathrm{D})\left(\begin{array}{c}
1 \\
\bar{\zeta}
\end{array}\right) \mathrm{d} \bar{\zeta}=\left(\begin{array}{c}
\boldsymbol{\nabla} \cdot(h \mathbf{u}) \\
\partial_{x}\left(h u^{2}+P(h)\right)+\partial_{y}(h u v) \\
\partial_{x}(h u v)+\partial_{y}\left(h v^{2}+P(h)\right)
\end{array}\right), \\
\int-\nabla_{\zeta} \cdot\left[\left(\mathrm{F}^{-2} \nabla h_{b}\right) \mathrm{D}\right]\left(\begin{array}{c}
1 \\
\bar{\zeta}
\end{array}\right) \mathrm{d} \bar{\zeta}=\left(\begin{array}{c}
0 \\
h \mathrm{~F}^{-2} \partial_{x} h_{b} \\
h \mathrm{~F}^{-2} \partial_{y} h_{b}
\end{array}\right)
\end{gathered}
$$

Combining expressions (A.3) with (A.2) confirms the proposition. Finally, we get $K=h^{2} / P(h)$ and $G=\sqrt{P(h) / h}$, since $K G^{2}=h$ and $K G^{4}=P(h)$.

\section{B Summary of exact solutions}

Exact solutions used in the verification tests of Section 4 are given next.

An exact solution of the Burgers' equation has been constructed by taking one of the Riemann invariants of the one-dimensional frictionless SWE to be constant. The solution of $u$ and $h$ can be related to the implicit solution $w=w(x, t)$ of the inviscid Burgers' equation before wave breaking. The exact solution for $h$ and $u$ is given by $h=((K-w) \mathrm{F} / 3)^{2}, \quad u=K-2 \mathrm{~F}^{-1} \sqrt{h}$, with $w(x, t)=w_{0}\left(x^{\prime}\right), x=x^{\prime}+w_{0}\left(x^{\prime}\right) t$ and $w(x, 0)=w_{0}(x)$ as initial condition. Wave breaking occurs at time $t_{b}=-1 / \min \left(d w_{0} / d x\right)$.

Assuming a steady state, Houghton and Kasahara [19] analyzed the onedimensional flow over a smooth convex obstacle. The solution to (2.3) and (2.4) is $h u=\mathcal{K}_{1}$ and $\mathrm{F}^{2} u^{2} / 2+\left(h+h_{b}\right)=\mathcal{K}_{2}$, where $\mathcal{K}_{1}$ and $\mathcal{K}_{2}$ are integration constants. Far away from the obstacle, it can be assumed that the non-dimensional velocity and water depth are both equal to one and the bed topography level is equal to zero. Therefore, $\mathcal{K}_{1}=1$ and $\mathcal{K}_{2}=\mathrm{F}^{2} / 2+1$. After some algebraic manipulations, the following third-order equation in $u$ is ob- 
tained $\mathrm{F}^{2} u^{3} / 2+\left(h_{b}-\mathrm{F}^{2} / 2-1\right) u+1=0$, which is solved to obtain the flow over a smooth convex obstacle.

Consider shallow water waves and bores in a channel. Nonlinear wave breaking is investigated by initializing the flow with a gravity wave solution of the linearized shallow water equations: $h(\mathbf{x}, t)=H+A \sin (l y) \sin (k x+\omega t)$, $u(\mathbf{x}, t)=-\frac{A \mathrm{~F}^{-2} k}{\omega} \sin (l y) \sin (k x+\omega t), v(\mathbf{x}, t)=\frac{A \mathrm{~F}^{-2} l}{\omega} \cos (l y) \cos (k x+\omega t)$ with amplitude $A$, frequency $\omega= \pm \sqrt{a^{2}\left(k^{2}+l^{2}\right)}, a^{2}=\mathrm{F}^{-2} H$, and $l=$ $(2 n+1) \pi / L_{y}, k=2 \pi m / L_{x}$. In the simulation, the following parameters were used $L_{x}=L_{y}=1.0, A=0.1, \mathrm{~F}=1, H=1, m=2$, and $n=0$.

\section{References}

[1] Akers B. Shallow water flow through a contraction. http://gfd.whoi.edu/proceedings/2005/PDFvol2005.html, 2005.

[2] Al-Tarazi M., Bokhove O, Kuipers J., Van Sint Annaland M., and Vreman A.W. Reservoir formation in shallow granular flows through a contraction. http://eprints.eemcs.utwente.nl/, 2005.

[3] Ambati V.R., and Bokhove O. Space-time discontinuous Galerkin finite element modeling of rotating shallow water flows. J. Comp. Phys., submitted, http://eprints.eemcs. utwente.nl/, 2006.

[4] Atkinson P.H., Westerink J.J., and Hervouet J.-M. Similarities between the quasi-bubble and the generalized wave continuity equation solutions to the shallow water equations. Int. J. Num. Meth. in Fluids 45 (2004), 689-714.

[5] Batten P., Clarke N., Lambert C., and Causon D.M. On the Choice of Wavespeeds for the HLLC Riemann Solver. SIAM J. Sci. Comput. 18 (1997) (6), 1552-1570.

[6] Bokhove O. Flooding and drying in finite-element Galerkin discretizations of shallow-water equations. Part I: One dimension. J. Sci. Comput. 22 (2005), 47-82.

[7] Bokhove O. Hamiltonian restriction of Vlasov equations into two-layer isopycnic and isentropic equations. Appl. Math. Lett. 18 (2005), 1418-1425.

[8] Bokhove O., Woods A.W., and Boer de A. Magma Flow through ElasticWalled Dikes. Theor. Comput. Fluid Dyn. 19 (2005), 261-286.

[9] Audusse E., Bouchut F., Bristeau M.-O., Klein R., Perthame B. A fast and stable well-balanced scheme with hydrostatic reconstruction for shallow water flows, SIAM J. Sci. Comp. 25, 2050-2065, 2004.

[10] Bühler O. On the vorticity transport due to dissipating or breaking waves in shallow-water flow. J. Fluid Mech. 407 (2000), 235-263.

[11] Castro M., Gallardo J.M., and Pares C. High order finite volume schemes based on reconstruction of states for solving hyperbolic systems with non- 
conservative products. Applications to shallow-water systems. Math. Comp. 75 (2006), 1103-1134.

[12] Chippada S., Dawson C.N., Martinez M.L., and Wheeler M.F. A Godunov-type finite volume method for the system of shallow water equations. Comp. Meth. Appl. Mech. and Eng. 151 (1998), 105-129.

[13] Cockburn B. and Shu C.-W. The Runge-Kutta discontinuous Galerkin finite element method for conservation laws V: Multidimensional systems. J. Comp. Phys. 141 (1998), pp. 199-224.

[14] Cockburn B. Discontinuous Galerkin methods for convection-dominated problems. In Timothy J. Barth and Herman Deconinck, editors, High-Order Methods for Computational Physics 9 (1999), pp. 69-224.

[15] Cockburn B., Karniadakis G.E., and Shu C.-W. The development of Discontinuous Galerking Methods. In C.-W. Shu B. Cockburn, G.E. Karniadakis, editor, Discontinuous Galerking Methods; Theory, Computation and Applications., volume 11 of Lecture Notes in Computational Science and Engineering. Springer, New York, Heidelberg, 2000.

[16] Flaherty J.E., R.M. Loy, M.S. Shepard, and J.D. Teresco. Software for the parallel adaptive solution of conservation laws by Discontinuous Galerkin Methods. In C.-W. Shu B. Cockburn, G.E. Karniadakis, editor, Discontinuous Galerkin Methods; Theory, Computation and Applications., volume 11 of Lecture Notes in Computational Science and Engineering. Springer, New York, Heidelberg, 2000.

[17] Hall P. Alternating bar instabilities in unsteady channel flows over erodible beds. J. Fluid Mech. 499 (2004), 49-73.

[18] Harten A., Lax P.D., and van Leer B. On upstream differencing and godunov-type schemes for hyperbolic conservation laws. SIAM Rev. 25 (1983), pp. 35-61.

[19] Houghton D. D. and Kasahara A. Nonlinear Shallow Fluid Flow Over an Isolated Ridge. Comm. on Pure and Applied Math. 21 (1968), 1-23.

[20] $\mathrm{Hu}, \mathrm{G}$. Note on numerical simulation of shallow water. Bristol University, School. of Mathematics. Rep. No. AM-02-01 (2002), 17 pp.

[21] Jaffre J., Johnson C., and Szepessy A. Convergence of the discontinuous Galerkin finite element method for hyperbolic conservation laws. Mathematical Models and Methods in Applied Sciences 5 (1995) (3), 367-386.

[22] Katopodes N.D. A dissipative Galerkin scheme for open-channel flows. J. Hydraul. Eng. ASCE 110 (1984), 450-466.

[23] Krivodonova L., Xin J., Remacle J.-F., and Chevaugeon N. Shock detection and limiting with discontinuous Galerkin methods for hyperbolic conservation laws. Applied Num. Math. 48 (2004), 323-338.

[24] Lamb J. Hydrodynamics. Cambridge University Press, 1993.

[25] Leer van, B. Towards the ultimate conservative difference scheme V: a second-order sequel to Godunov's method. J. Comp. Phys. 32 (1979), $361-370$.

[26] LeVeque, R.J. Numerical methods for conservation laws. Lectures in mathematics ETH Zürich, 1990. 
[27] Matsutomi, H. and Mochizula, A. Behaviour of a bore over a conical shoal. Tohoku Journal of Natural Disaster Science (2001) (in Japanese).

[28] Morrison P.J. The Maxwell-Vlasov equations as a continuous hamiltonian system. Phys. Lett. 80 (1980), 383-386.

[29] Pedlosky J. Geophysical Fluid Dynamics. Springer, 2nd edition, 1987.

[30] Peregrine D.H. Surf zone currents. Theor. Comput. Fluid Dyn. 10 (1998), 295-310.

[31] Peregrine, D.H. Large-scale vorticity generation in breakers in shallow and deep water. J. Mech. B/Fluids 18 (1999) 403-408.

[32] Perthame B. An introduction to kinetic schemes for gas dynamics. In Rohde C. Kröner D., Ohlberger M., editor, An introduction to recent developments in theory and numerics for conservation laws. Springer Verlag, pp. 1-27, 1999.

[33] Perthame B. and Simeoni C. A kinetic scheme for the Saint-Venant scheme with a source term. Calcolo 38 (2001), 201-231.

[34] Pratt L.J. On inertial flow over topography. Part 1. Semigeostrophic adjustment to an obstacle. J. Fluid Mech. 131 (1983), 195-218.

[35] Shu C.-W. TVD time discretizations. SIAM J. Sci. Stat. Comput. 9 (1988), 1073-1084.

[36] Tassi P., Bokhove O., and Vionnet, C. Additional appendices, http://eprints.eemcs.utwente.nl/, 2006.

[37] Toro, E. F. Riemann solvers and numerical methods for fluid dynamics : a practical introduction. Springer, 1997.

[38] Vegt van der, J.J.W. and Ven, van der H. Space-Time Discontinuous Galerkin Finite Element Method with Dynamic Grid Motion for Inviscid Compressible Flows: I. General Formulation. J. Comp. Phys. 182 (2002), 546-585.

[39] Vreugdenhil C.B. Numerical Methods for Shallow Water Flow. Kluwer Academic Publishers: Dordrecht, Netherlands, 2nd edition, 1994.

[40] Walters R.A. and Carey G.F. Analysis of spurious oscillations modes for the shallow-water and Navier-Stokes equations. Computers and Fluids 11 (1983) (2), 51-68.

[41] Whitham G.B. Linear and nonlinear waves. John Wiley, London., 1974.

[42] Zienkiewics O.C. and Ortiz P. A split-characteristic based finite element model for the shallow water equations. Int. J. Numer. Methods Fluids 20 (1995) (2), 1061-1080. 


\section{A Integral expressions in discretized kinetic formulation paper}

In the following, details about the implementation of the kinetic numerical flux are presented ${ }^{2}$. In (3.24)-(3.26), evaluation is required of the integrals

$$
\begin{aligned}
\left(\tilde{F}_{\sigma}^{\text {out }}\left(U^{-}\right), \tilde{F}_{\boldsymbol{\mu}}^{\text {out }}\left(U^{+}\right)\right) & =\int_{\hat{n}_{\mathrm{k}}^{-} \cdot \bar{\zeta}>0} \mathrm{D}^{-}\left(\hat{n}_{\mathrm{k}}^{-} \cdot \bar{\zeta}\right)(1, \bar{\zeta})^{T} \mathrm{~d} \bar{\zeta} \\
\left(\tilde{F}_{\sigma}^{\text {in }}\left(U^{+}\right), \tilde{F}_{\boldsymbol{\mu}}^{\text {in }}\left(U^{+}\right)\right) & =\int_{\hat{n}_{\mathrm{k}}^{-} \cdot \bar{\zeta} \leq 0} \mathrm{D}^{+}\left(\hat{n}_{\mathrm{k}}^{-} \cdot \bar{\zeta}\right)(1, \bar{\zeta})^{T} \mathrm{~d} \bar{\zeta}
\end{aligned}
$$

where

$$
\mathrm{D}=K(h) \chi((\bar{\xi}-\mathbf{u}) / G(h))
$$

In two dimensions a simple choice of $\chi(\bar{w})$ in (A.3) satisfying (3.18) is

$$
\chi(\bar{w})=\left\{\begin{array}{cc}
C_{\chi}=1 / 4 \pi & \text { if } \quad|\bar{w}|<R=2 \\
0 & \text { if } \quad|\bar{w}| \geq R
\end{array} .\right.
$$

The line $\hat{n}_{\mathrm{k}} \cdot \bar{\zeta}=\hat{n}_{\mathrm{k}} \cdot(G \bar{w}+\mathbf{u})=0$ demarcates the "left" and "right" integration regions in (A.1) and (A.2). Defining the distance $R_{I}=\left|\hat{n}_{\mathrm{k}} \cdot \mathbf{u}\right| / G$ of the origin in the $\bar{w}$-plane to the line $\hat{n}_{\mathrm{k}} \cdot \bar{\zeta}=0$, the following situations emerge considering the left region in which the domain of integration is $\hat{n}_{\mathrm{k}} \cdot \bar{\zeta}>0$ :

a) the line does not intersect the circle where $\chi(\cdot)$ jumps to zero, so $R_{I}>R$, and the circle lies outside the integration domain $\hat{n}_{\mathrm{k}} \cdot \bar{\zeta}>0$ since $\hat{n}_{\mathrm{k}} \cdot \mathbf{u}<0$, i.e. the origin $\bar{w}=0$ is excluded;

b) the line does not intersect the circle where $\chi(\cdot)$ jumps to zero, so $R_{I}>R$, and the circle lies inside the integration domain $\hat{n}_{\mathrm{k}} \cdot \bar{\zeta}>0$ since $\hat{n}_{\mathrm{k}} \cdot \mathbf{u} \geq 0$, i.e. the origin $\bar{w}=0$ is included; and

c1) circle and line intersect $R_{I} \leq R$ since $\hat{n}_{\mathrm{k}} \cdot \mathbf{u}<0$. i.e. the origin $\bar{w}=0$ is excluded; and

c2) circle and line intersect $R_{I} \leq R$ since $\hat{n}_{\mathrm{k}} \cdot \mathbf{u} \geq 0$, i.e. the origin $\bar{w}=0$ is included.

These situations are sketched in Fig. A.1.

For the left region, in situation a) the integrals are zero, and in situation b) the symmetry properties can be used. Vice versa, for the right region, in situation b) the integrals are zero, and in situation a) the symmetry properties can be used. Situation c) requires more attention. Rotating to new coordinates $\bar{w}^{\prime}$

$\overline{2}$ Appendix not included in the Advances in Water Resources. 
a)

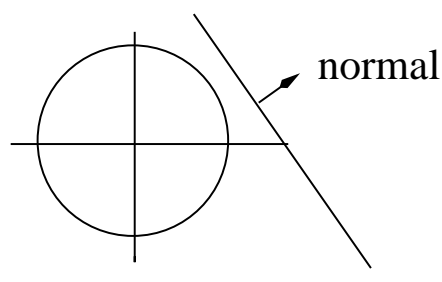

c1)

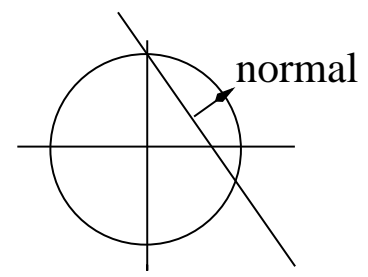

b)

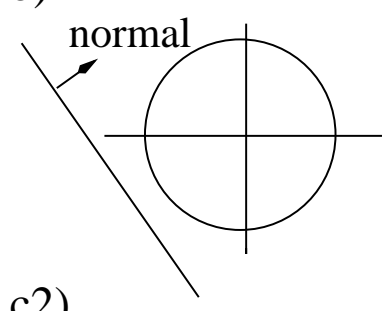

c2)

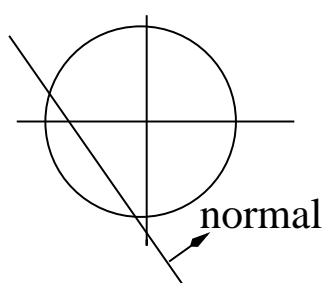

Fig. A.1. The circle with radius $R$ in (A.4) and the line $\hat{n}_{\mathrm{k}} \cdot \bar{\zeta}=0$ are sketched in the $\bar{w}$-plane. Situations at stake in the $\bar{w}^{\prime}$-plane: a) $R_{I}>R$ and $\hat{n}_{\mathrm{k}} \cdot \mathbf{u}<0$; b) $R_{I}>R$ and $\hat{n}_{\mathrm{k}} \cdot \mathbf{u} \geq 0$; c1) $R_{I} \leq R$ and $\hat{n}_{\mathrm{k}} \cdot \mathbf{u}<0$; and $\left.\mathrm{c} 2\right) R_{I} \leq R$ and $\hat{n}_{\mathrm{k}} \cdot \mathbf{u} \geq 0$.

such that the line intersects the positive $w_{1}^{\prime}$-axis only and runs parallel to the $w_{2}^{\prime}$-axis. In case $\left.\mathrm{c} 1\right)$, with $\hat{n}_{\mathrm{k}}=\left(n_{1}, n_{2}\right)$ the unit vectors of $\bar{w}^{\prime}$ are

$$
\hat{e}_{1}^{\prime}=\left(n_{1}, n_{2}\right)=\left(m_{1}, m_{2}\right) \quad \text { and } \quad \hat{e}_{2}^{\prime}=\left(-n_{2}, n_{1}\right)=\left(-m_{2}, m_{1}\right)
$$

In case $\mathrm{c} 2)$, with $\hat{n}_{\mathrm{k}}=\left(n_{1}, n_{2}\right)$ the unit vectors of $\bar{w}^{\prime}$ are

$$
\hat{e}_{1}^{\prime}=-\left(n_{1}, n_{2}\right)=\left(m_{1}, m_{2}\right) \quad \text { and } \quad \hat{e}_{1}^{\prime}=\left(n_{2},-n_{1}\right)=\left(-m_{2}, m_{1}\right) .
$$

Hence, the two coordinates can be related as follows

$$
w_{1}=m_{1} w_{1}^{\prime}-m_{2} w_{2}^{\prime} \quad \text { and } \quad w_{2}=m_{2} w_{1}^{\prime}+m_{1} w_{2}^{\prime} .
$$

Thus,

$$
\hat{n}_{\mathrm{k}} \cdot \bar{w}=\left(n_{1} m_{1}+n_{2} m_{2}\right) w_{1}^{\prime}+\left(-n_{1} m_{2}+n_{2} m_{1}\right) w_{2}^{\prime}=a_{1} w_{1}^{\prime}+a_{2} w_{2}^{\prime} .
$$

The following basic integrals in the $\bar{w}^{\prime}$-plane are calculated using xMaple under Linux

$$
\begin{aligned}
I_{0} & =C \int_{B} \mathrm{~d} \bar{w}^{\prime} \\
& =(1 /(4 \pi))\left(2 \pi-4\left(R_{I} / 2\right) \sqrt{1-\left(R_{I} / 2\right)^{2}}-4 \arcsin \left(R_{I} / 2\right)\right) \\
I_{1} & =C \int_{B} w_{1}^{\prime} \mathrm{d} \bar{w}^{\prime}=(4 /(3 \pi))\left(1-\left(R_{I} / 2\right)^{2}\right)^{3 / 2} \\
I_{2} & =C \int_{B} w_{2}^{\prime} \mathrm{d} \bar{w}^{\prime}=0
\end{aligned}
$$


and

$$
\begin{aligned}
I_{11} & =C \int_{B}\left(w_{1}^{\prime}\right)^{2} \mathrm{~d} \bar{w}^{\prime} \\
& =\left(2 \pi+\left(2 R_{I}-R_{I}^{3}\right) \sqrt{1-\left(R_{I} / 2\right)^{2}}-4 \arcsin \left(R_{I} / 2\right)\right) /(4 \pi) \\
I_{12} & =C \int_{B} w_{1}^{\prime} w_{2}^{\prime} \mathrm{d} \bar{w}^{\prime}=0 \\
I_{22} & =C \int_{B}\left(w^{\prime}{ }^{2}\right)^{2} \mathrm{~d} \bar{w}^{\prime} \\
& =\left(6 \pi+\left(-10 R_{I}+R_{I}^{3}\right) \sqrt{1-\left(R_{I} / 2\right)^{2}}-12 \arcsin \left(R_{I} / 2\right)\right) /(12 \pi)
\end{aligned}
$$

with $B=\left\{\bar{w}\left|w_{1}^{\prime}>R_{I},\right| \bar{w}^{\prime} \mid<R\right\}$ and $R_{I}<R$. In addition, by construction

$$
\begin{aligned}
& V_{1}=C \int_{B_{t}} w_{1}^{\prime} \mathrm{d} \bar{w}^{\prime}=V_{2}=C \int_{B_{t}} w_{2}^{\prime} \mathrm{d} \bar{w}^{\prime}=V_{12}=C \int_{B_{t}} w_{1}^{\prime} w_{2}^{\prime} \mathrm{d} \bar{w}^{\prime}=0 \\
& V_{0}=C \int_{B_{t}} \mathrm{~d} \bar{w}^{\prime}=V_{11}=C \int_{B_{t}}\left(w_{1}^{\prime}\right)^{2} \mathrm{~d} \bar{w}^{\prime}=V_{22}=C \int_{B_{t}}\left(w_{2}^{\prime}\right)^{2} \mathrm{~d} \bar{w}^{\prime}=1
\end{aligned}
$$

with $B_{t}=\left\{\bar{w}^{\prime}|| \bar{w}^{\prime} \mid<R\right\}$. Putting these results together, it can be found that

$$
\begin{aligned}
& F_{\sigma}^{\text {out }}=K^{-}\left(G^{-}\right)^{2}\left\{\begin{array}{cc}
0 & \text { a) } \\
\hat{n}_{\mathrm{k}} \cdot \mathbf{u}^{-} & \mathrm{b}) \\
G^{-} a_{1} I_{1}^{-}+\hat{n}_{\mathrm{k}} \cdot \mathbf{u}^{-} I_{0}^{-} & \mathrm{c} 1) \\
-G^{-} a_{1} I_{1}^{-}+\hat{n}_{\mathrm{k}} \cdot \mathbf{u}^{-}\left(V_{0}-I_{0}^{-}\right) & \mathrm{c} 2)
\end{array}\right. \\
& F_{\sigma}^{\text {in }}=K^{+}\left(G^{+}\right)^{2}\left\{\begin{array}{cc}
\hat{n}_{\mathrm{k}} \cdot \mathbf{u}^{+} & \mathrm{a}) \\
0 & \mathrm{~b}) \\
-G^{+} a_{1} I_{1}^{+}+\hat{n}_{\mathrm{k}} \cdot \mathbf{u}^{+}\left(V_{0}-I_{0}^{+}\right) & \mathrm{c} 1) \\
G^{+} a_{1} I_{1}^{+}+\hat{n}_{\mathrm{k}} \cdot \mathbf{u}^{+} I_{0}^{+} & \mathrm{c} 2)
\end{array}\right. \\
& \left(\begin{array}{rrr}
0 & \text { a) }
\end{array}\right. \\
& F^{\text {out }}=K^{-}\left(G^{-}\right)^{2} \quad\left(\hat{n}_{\mathrm{k}} \cdot \mathbf{u}\right) u_{j}^{-}+\hat{n}_{\mathrm{kj}}\left(G^{-}\right)^{2} \quad \text { b) } \\
& \left.F_{\mu_{(j)}}=K^{-}\left(G^{-}\right)^{2} \quad A_{11}^{(j)} I_{11}^{-}+A_{22}^{(j)} I_{22}^{-}+A_{1}^{(j)} I_{1}^{-}+A_{0}^{(j)} I_{0}^{-} \quad \mathrm{c} 1\right) \\
& \left.A_{11}^{(j)}\left(1-I_{11}^{-}\right)+A_{22}^{(j)}\left(1-I_{22}^{-}\right)-A_{1}^{(j)} I_{1}^{-}+A_{0}^{(j)}\left(1-I_{0}^{-}\right) \quad \mathrm{c} 2\right) \\
& F_{\mu_{(j)}}^{\mathrm{in}}=K^{+}\left(G^{+}\right)^{2}\left\{\begin{array}{c}
\left(\hat{n}_{\mathrm{k}} \cdot \mathbf{u}\right) u_{j}^{+}+\hat{n}_{\mathrm{kj}}\left(G^{+}\right)^{2} \\
0 \\
A_{11}^{(j)}\left(1-I_{11}^{+}\right)+A_{22}^{(j)}\left(1-I_{22}^{+}\right)-A_{1}^{(j)} I_{1}^{+}+A_{0}^{(j)}\left(1-I_{0}^{+}\right) \\
A_{11}^{(j)} I_{11}^{+}+A_{22}^{(j)} I_{22}^{+}+A_{1}^{(j)} I_{1}^{+}+A_{0}^{(j)} I_{0}^{+}
\end{array}\right.
\end{aligned}
$$


with

$a_{1}=n_{1} m_{1}+n_{2} m_{2}, \quad a_{2}=-n_{1} m_{2}+n_{2} m_{1}, \quad A_{0}^{(1)}=\left(\hat{n}_{\mathbf{k}} \cdot \mathbf{u}\right) u_{1}$,

$A_{1}^{(1)}=G\left[a_{1} u_{1}+\left(\hat{n}_{\mathrm{k}} \cdot \mathbf{u}\right) m_{1}\right], \quad A_{2}^{(1)}=G\left[a_{2} u_{1}-\left(\hat{n}_{\mathrm{k}} \cdot \mathbf{u}\right) m_{2}\right], \quad A_{11}^{(1)}=G^{2} a_{1} m_{1}$,

$A_{22}^{(1)}=-G^{2} a_{2} m_{2}, \quad A_{12}^{(1)}=G^{2}\left[-a_{1} m_{2}+a_{2} m_{1}\right], \quad A_{0}^{(2)}=\left(\hat{n}_{\mathrm{k}} \cdot \mathbf{u}\right) u_{2}$,

$A_{1}^{(2)}=G\left[a_{1} u_{2}+\left(\hat{n}_{\mathrm{k}} \cdot \mathbf{u}\right) m_{2}\right], \quad A_{2}^{(2)}=G\left[a_{2} u_{2}+\left(\hat{n}_{\mathrm{k}} \cdot \mathbf{u}\right) m_{1}\right], \quad A_{11}^{(2)}=G^{2} a_{1} m_{2}$,

$A_{22}^{(2)}=G^{2} a_{2} m_{1}, \quad A_{12}^{(2)}=G^{2}\left[a_{1} m_{1}+a_{2} m_{2}\right]$

evaluated with the proper trace value as is clear from each case but not explicitly denoted. Note that the expressions are consistent: When $R_{I}=R$, $I_{0,1,2}=0, I_{11}, I_{22}=0$ and cases c1,c2 reduce to cases a,b, respectively. When $R_{I}=0$ cases $\mathrm{c} 1$ and $\mathrm{c} 2$ give the same result, since $I_{0}=I_{11}=I_{22}=1 / 2$ and $I_{1}=4 /(3 \pi)$ and $\left(m_{1}, m_{2}\right)=\left(n_{1}, n_{2}\right)$ for case $\mathrm{c} 1$ and $\left(m_{1}, m_{2}\right)=-\left(n_{1}, n_{2}\right)$ for case $\mathrm{c} 2$. 